\title{
Analysis of the fictitious domain method with penalty for elliptic problems
}

\author{
Guanyu Zhou • Norikazu Saito
}

Received: 25 April 2012 / Revised: 3 April 2013 / Published online: 13 October 2013

(C) The JJIAM Publishing Committee and Springer Japan 2013

\begin{abstract}
The fictitious domain method with $H^{1}$-penalty for elliptic problems is considered. We propose a new way to derive the sharp error estimates between the solutions of original elliptic problems and their $H^{1}$-penalty problems, which can be applied to parabolic problem with moving-boundary maintaing the sharpness of the error estimate. We also prove some regularity theorems for $H^{1}$-penalty problems. The $P 1$ finite element approximation to $H^{1}$-penalty problems is investigated. We study error estimates between the solutions of $H^{1}$-penalty problems and discrete problems in $H^{1}$ norm, as well as in $L^{2}$ norm, which is not currently found in the literature. Thanks to regularity theorems, we can simplify the analysis of error estimates. Due to the integration on a curved domain, the discrete problem is not suitable for computation directly. Hence an approximation of the discrete problem is necessary. We provide an approximation scheme for the discrete problem and derive its error estimates. The validity of theoretical results is confirmed by numerical examples.
\end{abstract}

Keywords Fictitious domain methods · Finite element methods ·

Finite volume methods · Error bounds

Mathematics Subject Classification $\quad 65 \mathrm{M} 85 \cdot 76 \mathrm{M} 10 \cdot 76 \mathrm{M} 12 \cdot 65 \mathrm{~N} 15$

\section{Introduction}

The principle of the fictitious domain method is to solve the problem in a larger domain (the fictitious domain) containing the domain of interest with a very simple shape. Then,

G. Zhou $(\varangle) \cdot$ N. Saito

Graduate School of Mathematical Sciences, The University of Tokyo,

3-8-1 Komaba Meguro-ku, Tokyo 153-8914, Japan

e-mail: zhoug@ms.u-tokyo.ac.jp 
the fictitious domain is discretized by a uniform mesh, independent of the original boundary. The advantage of this approach is that we can avoid the time-consuming construction of a boundary-fitted mesh. One of these approaches is the penalty fictitious domain method which is based on a reformulation of the original problem in the fictitious domain by using a penalty parameter (see [2] for an introduction of other kinds of fictitious domain methods). In this article, we consider only the fictitious domain method with penalty. Obviously, the fictitious domain method is of use for time-dependent moving-boundary problems. Although there exist some ways to derive the sharp error estimates for elliptic problems (cf. $[8,11,12]$ ), it seems none of them has been applied to parabolic problem such that the sharpness of the error estimates are maintained. Our motivation lies in the study of the penalty fictitious domain method which can be applied to these time-dependent moving-boundary problems maintaining the sharpness of the error boundary. This is of obvious importance, and it seems that little is known in this direction. The fictitious domain method with penalty for parabolic problem firstly appeared in [6] to prove the existence of the solution for parabolic problem in time-dependent domain. Then, in [7], the convergence and finite difference approximation is given, but without error estimates. The $H^{1}$-penalty parabolic problem equals to a special interface problem, and in [1] the error estimate for elliptic and parabolic interface problem is studied. However, it is not so suitable to the $H^{1}$-penalty problem, and still, is only for time-independent domain when considering parabolic interface problem. As a primary step towards this final end, herein we examine some new methods of error analysis for elliptic problems that can be easily applied to parabolic problems in time-dependent domain with sharp error estimate (which has been presented in our another paper [14]). This is the purpose of this paper.

In order to illustrate our results, we consider the Dirichlet boundary value problem for the Poisson equation. The weak form $(Q)$ reads as

$$
\left\{\begin{array}{l}
\text { Find } u \in H_{0}^{1}(\Omega) \text { such that } \\
(\nabla u, \nabla v)_{\Omega}=(f, v)_{\Omega}, \quad \forall v \in H_{0}^{1}(\Omega)
\end{array}\right.
$$

where $\Omega \subset \mathbb{R}^{2}$ denotes a smooth bounded domain, $(\cdot, \cdot)_{\Omega}$ is the inner product of $L^{2}(\Omega)$ and $f \in L^{2}(\Omega)$. We can find a rectangular domain $D \supset \Omega, \Omega_{1}=D \backslash \bar{\Omega}$, and turn to solve the $H^{1}$-penalty problem $\left(Q_{\epsilon}\right)$ with penalty coefficient $0<\epsilon \ll 1$,

$$
\left\{\begin{array}{l}
\text { Find } u_{\epsilon} \in H_{0}^{1}(D) \text { such that } \\
\left(\nabla u_{\epsilon}, \nabla v\right)_{\Omega}+\frac{1}{\epsilon}\left(\nabla u_{\epsilon}, \nabla v\right)_{\Omega_{1}}=(\tilde{f}, v)_{D}, \quad \forall v \in H_{0}^{1}(D),
\end{array}\right.
$$

where $\tilde{f}$ is the zero extension of $f$ into $D$.

Another example of applying the fictitious domain method with penalty to (1.1) is,

$$
\left(\nabla u_{\epsilon}, \nabla v\right)_{\Omega}+\frac{1}{\epsilon}\left(u_{\epsilon}, v\right)_{\Omega_{1}}=(\tilde{f}, v), \forall v \in H_{0}^{1}(D),
$$

which we call the $L^{2}$-penalty problem. This is of interest; however, the $L^{2}$-penalty problem is beyond the scope of this paper, in which we shall concentrate our attention to the $H^{1}$-penalty problem. We have presented some results of $L^{2}$-penalty method in [15]. 
The error $\left\|u_{\epsilon}-u\right\|_{1, \Omega}\left(\|\cdot\|_{1, \Omega}=\|\cdot\|_{0, \Omega}+|\cdot|_{1, \Omega}\right)$ has been analyzed by many authors, where $\|\cdot\|_{1, \Omega}$ is the $H^{1}(\Omega)$ norm. In [7], it is bounded by $C \sqrt{\epsilon}(C$ is some constant, so as in the following), and in $[8,11,12]$ the sharp estimate $C \epsilon$ is achieved. In this paper, we give a new way to derive the sharp estimate. Moreover, we present some regularity analysis of $u_{\epsilon}$, which is useful for studying the $H^{1}$ and $L^{2}$ error between the solutions of $H^{1}$ problem $\left(Q_{\epsilon}\right)$ and its discrete problem, which is denoted as $\left(Q_{\epsilon, h}\right)$.

A Cartesian mesh can be introduced to the rectangular domain $D$ to get a uniformed triangulation $\mathcal{T}_{h}, h$ is the maximum diameter of the triangles of $\mathcal{T}_{h} . V_{h}(D)$ is the subspace of all piecewise linear continuous functions subordinate to $\mathcal{T}_{h}$. Then $\left(Q_{\epsilon, h}\right)$ reads as:

$$
\left\{\begin{array}{l}
\text { Find } u_{\epsilon, h} \in V_{h}(D) \text { such that } \\
\left(\nabla u_{\epsilon, h}, \nabla v_{h}\right)_{\Omega}+\frac{1}{\epsilon}\left(\nabla u_{\epsilon, h}, \nabla v_{h}\right)_{\Omega_{1}}=\left(\tilde{f}, v_{h}\right)_{D}, \quad \forall v_{h} \in V_{h}(D) .
\end{array}\right.
$$

Although there exist many works on finite element error estimate for elliptic problem with discontinuous coefficient or boundary unfitted mesh, we notice the discontinuous coefficient of $H^{1}$-penalty problem is dependent on the parameter $\epsilon$, such that methods on those works may not be so suitable for our problem. And most of those are not easy to apply to parabolic problem. So, we give a new analysis of the error estimate.

In the literature, there are several works devoted to the study of the $H^{1}$ error between $u_{\epsilon, h}$ and $u_{\epsilon}$ in $\Omega$. For example, in [12], it is proved that the $H^{1}$ error is bounded by $C(\epsilon+\epsilon \sqrt{h}+h \sqrt{\epsilon}+\sqrt{h})$. In our work, we prove a similar result with the analysis with a much simpler method of the analysis. The analysis of [12] is to consider the estimate of $u_{\epsilon, h}-u^{0}-\epsilon u^{1}$ to derive the final estimate of $u_{\epsilon}-u_{\epsilon, h}$, where $u^{0}$ is the zero extension of $u$, and $u^{1}$ is the solution of problem:

$$
-\Delta u^{1}=0 \text { in } \Omega_{1}, \quad \frac{\partial u^{1}}{\partial n^{-}}=\frac{\partial u^{0}}{\partial n}, \quad \text { and } u^{1}=0 \text { on } \partial D
$$

Here, $n$ is the unit outward normal to $\Gamma$ viewed as a boundary of $\Omega$, and $n^{-}$is opposite to $n$. We found the analysis in [12] to be complicated and not directly. Our method for estimating of $u_{\epsilon}-u_{\epsilon, h}$ is simpler, which is to find some interpolation of $u_{\epsilon}$, denoted as $v_{h}$, and then estimate $u_{\epsilon}-v_{h}$ by using a regularity theorem of $\left(Q_{\epsilon}\right)$.

Moreover, we show the $L^{2}$ error is bounded by $C(\epsilon+h+\sqrt{\epsilon h})$. A similar result of $H^{1}$ error for the elliptic problem in a specific domain is given in [8].

In discrete problem $\left(Q_{\epsilon, h}\right)$, we notice that we have to calculate the inner-product in a curved domain, for example, $\left(\nabla u_{\epsilon, h}, \nabla v_{h}\right)_{\Omega}$. So the discrete problem cannot be directly computed. We find that few prior works have provided a sufficient discussion on this issue; however, it is necessary to give an approximation scheme for solving $\left(Q_{\epsilon, h}\right)$ and the associated error estimates when applying the finite element method to computation.

Herein, we present an approximation scheme, that is, instead of solving $\left(Q_{\epsilon, h}\right)$ we solve some problem $\left(\hat{Q}_{\epsilon, h}\right)$ approximating to $\left(Q_{\epsilon, h}\right)$. $\left(\hat{Q}_{\epsilon, h}\right)$ reads as:

$$
\left\{\begin{array}{l}
\text { Find } \hat{u}_{\epsilon, h} \in V_{h}(D) \text { such that } \\
\left(\nabla \hat{u}_{\epsilon, h}, \nabla v_{h}\right)_{\hat{\Omega}}+\frac{1}{\epsilon}\left(\nabla \hat{u}_{\epsilon, h}, \nabla v_{h}\right)_{\hat{\Omega}_{1}}=\left(f_{h}, v_{h}\right)_{D}, \quad \forall v_{h} \in V_{h}(D),
\end{array}\right.
$$


where $\hat{\Omega}$ is a polygon approximating to $\Omega$, and $f_{h}$ is some interpolation of $\tilde{f}$. With assumptions that $\left\|f_{h}-f\right\|_{0, D} \leq C h, \hat{\Omega}$ is convex and $(\hat{\Omega} \backslash \Omega) \cup(\Omega \backslash \hat{\Omega}) \subset \Gamma_{\delta}, \delta=$ $O\left(h^{2}\right)$, where $\Gamma_{\delta}=\left\{x \in \mathbb{R}^{2} \mid \operatorname{dist}(x, \Gamma=\partial \Omega) \leq \delta\right\}$, we find that the error $\hat{u}_{\epsilon, h}-u_{\epsilon, h}$ is bounded by $C h$ in $H^{1}$ norm.

In above, we have restricted our attention to Dirichlet boundary problem. For Neumann and mixed boundary problems we also consider the approximation of $H^{1}$-penalty problems. Further, for Neumann boundary problem, the discrete problem is investigated. Although some results we obtain are similar to those of [12], as we mentioned in the beginning, our work is focus on solving parabolic problems with time-dependent domain, and all methods of analysis are applicable to this class of problems.

The rest of this paper is organized as follows. The $H^{1}$-penalty problems for original problems with Dirichlet, Neumann, and mixed boundaries are given in Sect. 2, as well as the analysis of error estimates between the solutions of original problems and $H^{1}$ penalty problems, in a different way from that in $[8,11]$.

In Sect. 3, we present some regularity theorems for $H^{1}$-penalty problems. The $H^{1}$ penalty problem is in a sense equivalent to a kind of interface problem. The regularity theorem for the interface problem has been studied in [9]. However, we make several improvements in priori estimates and identify some higher-order regularity for our problems. The theorems will be used in Sect. 4 to make the error estimate more simple than that of [12].

The Sect. 4 is devoted to discrete problems. Finite element approximations are investigated. Using the same separation method of the triangulated domain as in [12], regularity theorems in Sect. 3 and some lemmas from $[3,10]$, we obtain the error estimates in $H^{1}$ norm with the same order as in $[8,12]$. Moreover, we give the higherorder $L^{2}$ norm error estimates.

We consider a scheme approximating to the discrete problem in Sect. 5. We introduce a new discrete problem $\left(\hat{Q}_{\epsilon, h}\right)$ to approximate to the discrete problem $\left(Q_{\epsilon, h}\right)$. Of necessity, due to insufficient prior reported works on this issue in the literature, we derive some error estimates of the scheme to make the numerical analysis of the fictitious domain method with $H^{1}$-penalty more complete.

Finally, we give some numerical experiments to verify our theoretical results in Sect. 6.

\section{$2 H^{1}$-penalty problems of fictitious domain method for elliptic problems}

Following the notation given in the previous section, we state the $H^{1}$-penalty problem for the original elliptic problem with homogeneous Dirichlet, Neumann and mixed boundary conditions. In addition, we write $\Gamma=\partial \Omega$.

\subsection{Dirichlet boundary value problem}

First, we consider the Dirichlet boundary value problem (1.1) and its $H^{1}$-penalty problem (1.2).

Theorem 2.1 There exist unique solutions $u$ and $u_{\epsilon}$ for (1.1) and (1.2), respectively, and we have the following estimates: 


$$
\begin{aligned}
& \left\|u_{\epsilon}-u\right\|_{1, \Omega} \leq C \epsilon, \\
& \left\|u_{\epsilon}\right\|_{1, \Omega_{1}} \leq C \epsilon .
\end{aligned}
$$

Those error estimates themselves are not new: they have been stated in [8] and [11]. The main process to prove those estimates in [8] and [11] are different (see Remark 3 below). We shall give a somewhat new proof which will be used for parabolic problems. Before stating that, we recall the well-known extension and trace theorems that we frequently use.

Lemma 2.2 (Theorem 8.1 of Chapter 1 in [5]) Let $\omega \subset \mathbb{R}^{2}$ be a bounded domain with the smooth boundary $\partial \omega$. Then, for any integer $k>0$, there exists an operator $E_{k}(\omega): H^{k}(\omega) \rightarrow H^{k}\left(\mathbb{R}^{2}\right)$ with the properties

$$
\begin{aligned}
& E_{k}(\omega) u=u \text { a.e. on } \omega\left(u \in H_{k}(\omega)\right), \\
& \left\|E_{k}(\omega) u\right\|_{H^{k}\left(\mathbb{R}^{2}\right)} \leq C_{k}\|u\|_{H^{k}(\omega)}\left(u \in H_{k}(\omega)\right),
\end{aligned}
$$

with a domain constant $C_{k}>0$.

The following lemma is a readily obtainable consequence of Theorem 8.3 of Chapter 1 in [5].

Lemma 2.3 Let $\omega \subset \mathbb{R}^{2}$ be a bounded domain. Assume that the boundary $\partial \omega$ consists of two disjoint and smooth components $\partial \omega_{1}$ and $\partial \omega_{2} ; \partial \omega=\partial \omega_{1} \cup \partial \omega_{2}$. Then, the mapping $\left.u \mapsto u\right|_{\partial \omega_{1}}$ of $C^{\infty}(\bar{\omega}) \rightarrow C^{\infty}\left(\partial \omega_{1}\right)$ is extended by continuity to a continuous linear mapping, which is called the trace operator and denoted by $\gamma\left(\omega, \partial \omega_{1}\right)$, of $H^{1}(\omega) \rightarrow H^{\frac{1}{2}}\left(\partial \omega_{1}\right)$. This mapping is surjective and there exists a continuous linear right inverse, which is called the lifting operator, $g \mapsto \gamma\left(\omega, \partial \omega_{1}\right)^{-1} g$ of $H^{\frac{1}{2}}\left(\partial \omega_{1}\right) \rightarrow$ $H^{1}(\omega)$ such that $\gamma\left(\omega, \partial \omega_{1}\right) \gamma\left(\omega, \partial \omega_{1}\right)^{-1} g=g$.

Remark 1 In view of Lemma 2.2, there exists an operator $E_{1,0}(\Omega): H^{1}(\Omega) \rightarrow$ $H_{0}^{1}(D)$ with properties:

$$
\begin{aligned}
& E_{1,0}(\Omega) u=u \text { a.e. in } \Omega\left(u \in H^{1}(\Omega)\right), \\
& \left\|E_{1,0}(\Omega) u\right\|_{H^{1}(D)} \leq C\|u\|_{H^{1}(\Omega)}\left(u \in H^{1}(\Omega)\right) .
\end{aligned}
$$

In fact, taking $\phi \in C_{0}^{\infty}(D)$ with $0 \leq \phi \leq 1$ and $\phi=1$ in $\Omega^{\prime}$, where $\Omega \subset \Omega^{\prime} \subset D$, then the desired operator is defined as $E_{1,0}(\Omega) u=\phi \cdot E_{1}(\Omega) u$ for $u \in H^{1}(\Omega)$.

Now, we can state the following proof.

Proof of Theorem 2.1 Firstly, by the Lax-Milgram Theorem, the unique existence of $u$ and $u_{\epsilon}$ is obvious. And we can obtain the estimate for the solution of the $H^{1}$-penalty problem (1.2),

$$
\left\|u_{\epsilon}\right\|_{1, D} \leq C\|f\|_{0} \inf \left(1, \frac{1}{\epsilon}\right) .
$$


Without loss of generality, we assume $0<\epsilon \leq 1$ in what follows. Substituting $v=E_{1,0}\left(\Omega_{1}\right) u_{\epsilon} \in H_{0}^{1}(D)$ into (1.2), we obtain

$$
\epsilon\left(\nabla u_{\epsilon}, v\right)_{\Omega}+\left\|\nabla u_{\epsilon}\right\|_{0, \Omega_{1}}^{2}=\epsilon(\tilde{f}, v)_{D}
$$

which leads to an estimate of $\left.u_{\epsilon}\right|_{\Omega_{1}}$, in particular,

$$
C_{1}\left\|u_{\epsilon}\right\|_{1, \Omega_{1}}^{2} \leq\left\|\nabla u_{\epsilon}\right\|_{0, \Omega_{1}}^{2} \leq C \epsilon\|f\|_{0, \Omega}\|v\|_{1, D}+\epsilon\left|u_{\epsilon}\right|_{1, \Omega}|v|_{1, \Omega}
$$

Therein, the first inequality is deduced by Friedrichs' inequality, and the second term of the right-hand side is bounded by $C_{2} \epsilon\|f\|_{0}\left\|u_{\epsilon}\right\|_{1, \Omega_{1}}$. Thus we have

$$
\left\|u_{\epsilon}\right\|_{1, \Omega_{1}} \leq C \epsilon
$$

Next, we consider the trace operators

$$
\gamma(\Omega, \Gamma): H^{1}(\Omega) \rightarrow H^{\frac{1}{2}}(\Gamma) ; \gamma\left(\Omega_{1}, \Gamma\right): H^{1}\left(\Omega_{1}\right) \rightarrow H^{\frac{1}{2}}(\Gamma)
$$

Since $u_{\epsilon} \in H^{1}(D)$, we have $\gamma(\Omega, \Gamma) u_{\epsilon}=\gamma\left(\Omega_{1}, \Gamma\right) u_{\epsilon} \in H^{\frac{1}{2}}(\Gamma)$. Hence,

$$
\gamma(\Omega, \Gamma)\left(u-u_{\epsilon}\right)=\gamma(\Omega, \Gamma) u-\gamma(\Omega, \Gamma) u_{\epsilon}=0-\gamma\left(\Omega_{1}, \Gamma\right) u_{\epsilon} .
$$

Setting $w=u-\left.u_{\epsilon}\right|_{\Omega}$, we have

$$
\|\gamma(\Omega, \Gamma) w\|_{\frac{1}{2}, \Gamma}=\left\|\gamma\left(\Omega_{1}, \Gamma\right) u_{\epsilon}\right\|_{\frac{1}{2}, \Gamma} \leq C\left\|u_{\epsilon}\right\|_{1, \Omega_{1}} \leq C \epsilon
$$

We define an operator $A: H^{1}(\Omega) \rightarrow H^{-1}(\Omega)$ by $\langle A u, v\rangle=(\nabla u, \nabla v)_{\Omega}, \forall v \in$ $H_{0}^{1}(\Omega)$. We observe that for any $v \in H_{0}^{1}(\Omega)$,

$$
\begin{aligned}
\langle A w, v\rangle & =(\nabla u, \nabla v)_{\Omega}-\left(\nabla u_{\epsilon}, \nabla v\right)_{\Omega}=(\nabla u, \nabla v)_{\Omega}-\left(\nabla u_{\epsilon}, \nabla \tilde{v}\right)_{\Omega} \\
& =(f, v)_{\Omega}-(\tilde{f}, \tilde{v})_{D}+\frac{1}{\epsilon}\left(\nabla u_{\epsilon}, \nabla \tilde{v}\right)_{\Omega_{1}} \\
& =(f, v)_{\Omega}-(f, v)_{\Omega}+\frac{1}{\epsilon}\left(\nabla u_{\epsilon}, 0\right)_{\Omega_{1}}=0,
\end{aligned}
$$

where $\tilde{v}$ means the zero extension of $v$ into D. This implies $A w=0$ in $H^{-1}(\Omega)$.

Since $w \rightarrow\{A w, \gamma(\Omega, \Gamma) w\}$ is an isomorphic map of $H^{1}(\Omega) \rightarrow H^{-1}(\Omega) \times$ $H^{\frac{1}{2}}(\Gamma)$, we obtain that $\left\|u-u_{\epsilon}\right\|_{1, \Omega}=\|w\|_{1, \Omega} \leq C\left(\|A w\|_{H^{-1}(\Omega)}+\|\gamma(\Omega, \Gamma) w\|_{\frac{1}{2}, \Gamma}\right) \leq C(0+C \epsilon) \leq C \epsilon$ which completes the proof.

Remark 2 The conclusion of Theorem 2.1 remains valid even for $f \in H^{-1}(\Omega)$. 
Remark 3 The saddle-point method in [8] requires a symmetric variational form, and the operator method in [11] is not so easy to deal with the operator of time-derivative when one considers parabolic problems. However, our method of analysis is applicable to parabolic problems; this is a recent achievement, and will be presented in our future work.

\subsection{Neumann boundary value problem}

The original problem $(Q)$ with homogeneous Neumann boundary condition reads as:

$$
\left\{\begin{array}{l}
\text { Find } u \in H^{1}(\Omega) \text { such that } \\
(u, v)_{1, \Omega}=(f, v)_{\Omega}, \quad \forall v \in H^{1}(\Omega)
\end{array}\right.
$$

where $(u, v)_{1, \Omega}:=(\nabla u, \nabla v)_{\Omega}+(u, v)_{\Omega}$.

The $H^{1}$-penalty problem $\left(Q_{\epsilon}\right)$ reads as:

$$
\left\{\begin{array}{l}
\text { Find } u_{\epsilon} \in H_{0}^{1}(D) \text { such that } \\
\left(u_{\epsilon}, v\right)_{1, \Omega}+\epsilon\left(\nabla u_{\epsilon}, \nabla v\right)_{\Omega_{1}}=(\tilde{f}, v)_{D}, \forall v \in H_{0}^{1}(D)
\end{array}\right.
$$

Theorem 2.4 There exist unique solutions $u$ and $u_{\epsilon}$ for (2.3) and (2.4), respectively, and we have the following estimates:

$$
\begin{aligned}
& \left\|u_{\epsilon}-u\right\|_{1, \Omega} \leq C \epsilon, \\
& \left\|u_{\epsilon}\right\|_{1, \Omega_{1}} \leq C .
\end{aligned}
$$

Proof Firstly, substituting $v=u_{\epsilon}$ into (2.4), we obtain

$$
\left\|u_{\epsilon}\right\|_{1, \Omega}^{2}+\epsilon\left\|\nabla u_{\epsilon}\right\|_{\Omega_{1}}^{2}=\left(f, u_{\epsilon}\right)_{\Omega}
$$

This gives

$$
\left\|u_{\epsilon}\right\|_{1, \Omega} \leq C\|f\|_{\Omega} \text {. }
$$

Since $v \in H_{0}^{1}(D)$ implies $\left.v\right|_{\Omega} \in H^{1}(\Omega)$, subtracting (2.3) from (2.4), we have

$$
\left(u_{\epsilon}-u, v\right)_{1, \Omega}+\epsilon\left(\nabla u_{\epsilon}, \nabla v\right)_{\Omega_{1}}=0, \forall v \in H_{0}^{1}(D) .
$$

Setting $v=u_{\epsilon}-E_{1,0}(\Omega) u_{\epsilon}$ in (2.7), and noticing that $\left.v\right|_{\Omega}=0$, we have

$$
\left(u_{\epsilon}-u, 0\right)_{1, \Omega}+\epsilon\left(\nabla u_{\epsilon}, \nabla\left(u_{\epsilon}-E_{1,0}(\Omega) u_{\epsilon}\right)\right)_{\Omega_{1}}=0 .
$$


And we see that

$$
\begin{aligned}
& \left\|\nabla u_{\epsilon}\right\|_{\Omega_{1}}^{2}=\left(\nabla u_{\epsilon}, \nabla E_{1,0}(\Omega) u_{\epsilon}\right)_{\Omega_{1}} \\
\leq & \left\|\nabla u_{\epsilon}\right\|_{\Omega_{1}}\left\|\nabla E_{1,0}(\Omega) u_{\epsilon}\right\|_{\Omega_{1}} \leq\left\|\nabla u_{\epsilon}\right\|_{\Omega_{1}}\left\|E_{1,0}(\Omega) u_{\epsilon}\right\|_{1, D} \\
\leq & C\left\|\nabla u_{\epsilon}\right\|_{\Omega_{1}}\left\|u_{\epsilon}\right\|_{1, \Omega} \leq C\left\|\nabla u_{\epsilon}\right\|_{\Omega_{1}}\|f\|_{\Omega} .
\end{aligned}
$$

Together with Friedrichs' inequality, we have

$$
\left\|u_{\epsilon}\right\|_{1, \Omega_{1}} \leq C\left\|\nabla u_{\epsilon}\right\|_{\Omega_{1}} \leq C\|f\|_{\Omega} .
$$

Thus, we proved (2.6).

In order to derive (2.5), we substitute $v=E_{1,0}(\Omega)\left(\left.u_{\epsilon}\right|_{\Omega}-u\right) \in H_{0}^{1}(D)$ in (2.7). Then, together with (2.6), we have

$$
\left\|u_{\epsilon}-u\right\|_{1, \Omega}^{2}=-\epsilon\left(\nabla u_{\epsilon}, \nabla v_{1}\right)_{\Omega_{1}} \leq C \epsilon\left|v_{1}\right|_{1, \Omega_{1}} \leq C_{1} \epsilon\left\|\left.u_{\epsilon}\right|_{\Omega}-u\right\|_{1, \Omega}
$$

which implies (2.5). We complete the proof.

Remark 4 Recall Remark 3 again. It should be noticed that our analysis method is much simpler than that in $[8,11]$.

\subsection{Mixed boundary value problem}

Let $\Gamma=\Gamma_{1} \cup \Gamma_{2}, \Gamma_{1} \cap \Gamma_{2}=\emptyset$. The domian $D \backslash \bar{\Omega}$ is assumed to be split into two part $\Omega_{1}$ and $\Omega_{2}$, which respectively share the boundaries $\Gamma_{1}$ and $\Gamma_{2}$ with $\Omega$. In addition both share non-empty measure boundary with $D$ (see Fig. 1). Set

$$
V=\left\{u \in H^{1}(\Omega) \mid \gamma\left(\Omega, \Gamma_{1}\right) u=0\right\} .
$$

The original problem $(Q)$ with homogeneous mixed boundary is stated as:

$$
\left\{\begin{array}{l}
\text { Find } u \in V \text { such that } \\
(\nabla u, \nabla v)_{\Omega}=(f, v)_{\Omega}, \forall v \in V
\end{array}\right.
$$

The $H^{1}$-penalty problem $\left(Q_{\epsilon}\right)$ reads as:

$$
\left\{\begin{array}{l}
\text { Find } u_{\epsilon} \in H_{0}^{1}(D) \text { such that } \\
\left(\nabla u_{\epsilon}, \nabla v\right)_{\Omega}+\frac{1}{\epsilon}\left(\nabla u_{\epsilon}, \nabla v\right)_{\Omega_{1}} \\
+\epsilon\left(\nabla u_{\epsilon}, \nabla v\right)_{\Omega_{2}}=(\tilde{f}, v), \forall v \in H_{0}^{1}(D)
\end{array}\right.
$$

Theorem 2.5 There exist unique solutions $u$ and $u_{\epsilon}$ for (2.8) and (2.9), respectively, and we have the following estimates:

$$
\left\|u_{\epsilon}-u\right\|_{1, \Omega} \leq C \epsilon
$$


Fig. 1 On mixed boundary case

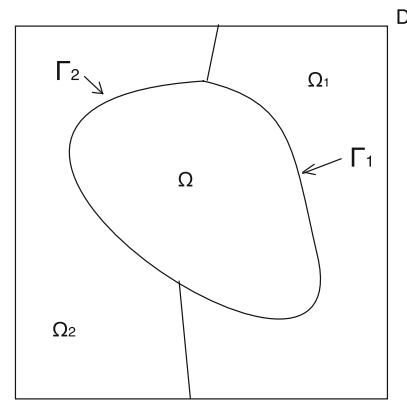

$$
\begin{aligned}
& \left\|u_{\epsilon}\right\|_{1, \Omega_{1}} \leq C \epsilon, \\
& \left\|u_{\epsilon}\right\|_{1, \Omega_{2}} \leq C .
\end{aligned}
$$

Proof The following results have already been achieved (Theorem I-8 in [7]):

$$
\begin{array}{rll}
\left.u_{\epsilon}\right|_{\Omega} \rightarrow u & \text { in } & H^{1}(\Omega) ; \\
\frac{\left.u_{\epsilon}\right|_{\Omega_{1}}}{\sqrt{\epsilon}} \rightarrow 0 & \text { in } & H^{1}\left(\Omega_{1}\right) ; \\
\left.\sqrt{\epsilon} u_{\epsilon}\right|_{\Omega_{2}} \rightarrow 0 & \text { in } & H^{1}\left(\Omega_{2}\right),
\end{array}
$$

as $\epsilon \rightarrow 0$. Noting $\Omega^{*}=\Omega \cup \Omega_{1} \cup \Gamma_{1}$, we set $v=u_{\epsilon}-E_{1,0}\left(\Omega^{*}\right) u_{\epsilon} \in H_{0}^{1}(D)$. From (2.13) and (2.14), we have

$$
\left\|E_{1,0}\left(\Omega^{*}\right) u_{\epsilon}\right\|_{1, D} \leq C\left\|u_{\epsilon}\right\|_{1, \Omega^{*}} \leq C .
$$

We find that $\left.v\right|_{\Omega^{*}}=0$ and substitute this $v$ into (2.9) to obtain

$$
\epsilon\left(\nabla u_{\epsilon}, \nabla v\right)_{\Omega_{2}}=0
$$

This gives

$$
\left|u_{\epsilon}\right|_{1, \Omega_{2}}^{2}=\left(\nabla u_{\epsilon}, \nabla E_{1,0}\left(\Omega^{*}\right) u_{\epsilon}\right)_{\Omega_{2}} \leq\left|u_{\epsilon}\right|_{1, \Omega}\left\|E_{1,0}\left(\Omega^{*}\right) u_{\epsilon}\right\|_{1, D} .
$$

Combining with $\left\|u_{\epsilon}\right\|_{1, \Omega_{2}} \leq C\left|u_{\epsilon}\right|_{1, \Omega_{2}}$ and (2.16), it shows (2.12).

Substitueting $v=E_{1,0}\left(\Omega_{1}\right) u_{\epsilon}$ into (2.9), we have

$$
\left(\nabla u_{\epsilon}, \nabla E_{1,0}\left(\Omega_{1}\right) u_{\epsilon}\right)_{\Omega}+\frac{1}{\epsilon}\left\|\nabla u_{\epsilon}\right\|_{\Omega_{1}}^{2}+\epsilon\left(\nabla u_{\epsilon}, \nabla E_{1,0}\left(\Omega_{1}\right) u_{\epsilon}\right)_{\Omega_{2}}=\left(\tilde{f}, E_{1,0}\left(\Omega_{1}\right) u_{\epsilon}\right),
$$

from which we obtain that

$$
\begin{aligned}
& \left\|u_{\epsilon}\right\|_{1, \Omega_{1}}^{2} \leq C\left|u_{\epsilon}\right|_{1, \Omega_{1}}^{2} \\
= & C\left(\epsilon\left(\nabla u_{\epsilon}, \nabla E_{1,0}\left(\Omega_{1}\right) u_{\epsilon}\right)_{\Omega}+\epsilon^{2}\left(\nabla u_{\epsilon} \cdot \nabla E_{1,0}\left(\Omega_{1}\right) u_{\epsilon}\right)_{\Omega_{2}}+\epsilon\left(\tilde{f}, E_{1,0}\left(\Omega_{1}\right) u_{\epsilon}\right)\right) \\
\leq & C \epsilon\left(\|f\|_{\Omega}+\left|u_{\epsilon}\right|_{1, \Omega}+\epsilon\left|u_{\epsilon}\right|_{1, \Omega_{2}}\right)\left\|E_{1,0}\left(\Omega_{1}\right) u_{\epsilon}\right\|_{1, D} \leq C \epsilon\left\|u_{\epsilon}\right\|_{1, \Omega_{1}},
\end{aligned}
$$


which implies (2.11).

Setting $w=\left.u_{\epsilon}\right|_{\Omega}-u$, we have $\gamma\left(\Omega, \Gamma_{1}\right) w=\gamma\left(\Omega, \Gamma_{1}\right) u_{\epsilon}-0=\gamma\left(\Omega_{1}, \Gamma_{1}\right) u_{\epsilon}$, which shows

$$
\left\|\gamma\left(\Omega, \Gamma_{1}\right) w\right\|_{\frac{1}{2}, \Gamma_{1}} \leq C\left\|u_{\epsilon}\right\|_{1, \Omega_{1}} \leq C \epsilon
$$

Applying Lemma 2.3, there exists $w_{1}=\gamma\left(\Omega, \Gamma_{1}\right)^{-1} \gamma\left(\Omega, \Gamma_{1}\right) w \in H^{1}(\Omega)$, satisfying

$$
\left\|w_{1}\right\|_{1, \Omega} \leq C\left\|\gamma\left(\Omega, \Gamma_{1}\right) w\right\|_{\frac{1}{2}, \Gamma_{1}} \leq C \epsilon
$$

Next, we define a continuous operator $\bar{A}: V \rightarrow V^{\prime}\left(V^{\prime}\right.$ is the dual space of $V$ ) by $\langle\bar{A} \phi, v\rangle=(\nabla \phi, \nabla v)_{\Omega}, \phi \in V, \forall v \in V$. Applying Friedrichs' inequality, we have

$$
(\nabla v, \nabla v)_{\Omega}=|v|_{1, \Omega}^{2} \geq C\|v\|_{1, \Omega}^{2}=\|v\|_{V}^{2}
$$

Hence, the bilinear form $a(\phi, v)$ on $V \times V$ defined by $a(\phi, v)=\langle\bar{A} \phi, v\rangle$ is $V$-elliptic. It follows from the Lax-Milgram theorem that

$$
\left\|w-w_{1}\right\|_{V} \leq C\left\|\bar{A}\left(w-w_{1}\right)\right\|_{V^{\prime}} \leq C\left(\|\bar{A} w\|_{V^{\prime}}+\left\|\bar{A} w_{1}\right\|_{V^{\prime}}\right)
$$

As $\left\|u_{\epsilon}-u\right\|_{1, \Omega}=\|w\|_{V} \leq\left\|w-w_{1}\right\|_{V}+\left\|w_{1}\right\|_{V} \leq\left\|w-w_{1}\right\|_{V}+C \epsilon$, to prove (2.10) we need to show that $\left\|w-w_{1}\right\|_{V} \leq C \epsilon$, where we already know $\left\|\bar{A} w_{1}\right\|_{V^{\prime}} \leq$ $C\left\|w_{1}\right\|_{1, \Omega} \leq C \epsilon$ by the continuity of $\bar{A}$. To estimate $\|\bar{A} w\|_{V^{\prime}}$, we observe that $\forall v \in$ $V, \bar{v}=E_{1,0}(\Omega) v \in H_{0}^{1}(D)$ with $\left.E_{1,0}(\Omega) v\right|_{\Omega_{1}}=0$,

$$
\begin{aligned}
\langle\bar{A} w, v\rangle & =\left(\nabla u_{\epsilon}, \nabla v\right)_{\Omega}-(\nabla u, \nabla v)_{\Omega}=\left(\nabla u_{\epsilon}, \nabla \bar{v}\right)_{\Omega}-(\nabla u, \nabla v)_{\Omega} \\
& =(\tilde{f}, \bar{v})_{D}-\frac{1}{\epsilon}\left(\nabla u_{\epsilon}, \nabla \bar{v}\right)_{\Omega_{1}}-\epsilon\left(\nabla u_{\epsilon}, \nabla \bar{v}\right)_{\Omega_{2}}-(f, v)_{\Omega} \\
& =(f, v)_{\Omega}-\frac{1}{\epsilon}\left(\nabla u_{\epsilon}, 0\right)_{\Omega_{1}}-\epsilon\left(\nabla u_{\epsilon}, \nabla \bar{v}\right)_{\Omega_{2}}-(f, v)_{\Omega} \\
& \leq \epsilon\left|u_{\epsilon}\right|_{1, \Omega_{2}}|\bar{v}|_{1, \Omega_{2}} \\
& \leq C \epsilon\|v\|_{1, \Omega},
\end{aligned}
$$

that is $\|\bar{A} w\|_{V^{\prime}} \leq C \epsilon$. Thus, we have $\left\|w-w_{1}\right\|_{V} \leq C\left(\|\bar{A} w\|_{V^{\prime}}+\left\|\bar{A} w_{1}\right\|_{V^{\prime}}\right) \leq C \epsilon$, and the proof is completed.

Remark 5 Basically, the proof for the mixed boundary case is a combination of those for the Dirichlet and Neumann boundary cases.

Remark 6 All of the above in this section, which involve homogeneous boundary conditions are also suitable for the non-homogeneous boundary value problems. 


\section{The regularity of the solutions of $H^{1}$-penalty problems}

This section is devoted to the regularity theorems for $\left(Q_{\epsilon}\right)$ with homogeneous Dirichlet, Neumann, and mixed boundary conditions respectively. As it is shown in [7], these $\left(Q_{\epsilon}\right)$ are equal to certain interface elliptic problems, denoted as $\left(P_{\epsilon}\right)$. There are some regularity theorems for the interface elliptic problems in the literature (see [1,9] for example), however, these are not specific to our problems. Our particular objective is to deduce explicit dependence of various norms of $u_{\epsilon}$ on the penalty parameter $\epsilon$. We show some estimates which are only suitable for our problems, as well as some higher-order regularity which will be used in the study of the $H^{1}$-penalty parabolic problem.

\subsection{Dirichlet boundary value problem}

As a first step, let us assume $D$ is sufficiently smooth. Then, we have the following theorem.

Theorem 3.1 Let $u_{\epsilon} \in H_{0}^{1}(D)$ be the solution of the $H^{1}$-penalty problem (1.2) for $f \in L^{2}(\Omega)$. Then, we have

$$
\begin{aligned}
& \left.u_{\epsilon}\right|_{\Omega} \in H^{2}(\Omega),\left.u_{\epsilon}\right|_{\Omega_{1}} \in H^{2}\left(\Omega_{1}\right), \\
& \left\|u_{\epsilon}\right\|_{2, \Omega} \leq C\|f\|_{0, \Omega}, \\
& \left\|u_{\epsilon}\right\|_{2, \Omega_{1}} \leq C \epsilon\|f\|_{0, \Omega} .
\end{aligned}
$$

First, we recall the following basic regularity result:

Lemma 3.2 Let $\omega \subset \mathbb{R}^{2}$ be a bounded domain. Assume that the boundary $\partial \omega$ is divided into two disjoint smooth components $\partial \omega_{1}$ and $\partial \omega_{2} ; \partial \omega=\partial \omega_{1} \cup \partial \omega_{2}$. Let $v \in H^{1}(\omega)$ be the unique weak solution of

$$
\Delta v=f \text { in } \omega, v=g_{1} \text { on } \partial \omega_{1}, \frac{\partial v}{\partial n}=g_{2} \text { on } \partial w_{2},
$$

where $f \in L^{2}(\omega), g_{1} \in H^{\frac{1}{2}}\left(\partial \omega_{1}\right)$ and $g_{2} \in L^{2}\left(\partial \omega_{2}\right)$.

Then, if $g_{1} \in H^{\frac{3}{2}}\left(\partial \omega_{1}\right)$ and $g_{2} \in H^{\frac{1}{2}}\left(\partial \omega_{2}\right)$, we have $v \in H^{2}(\omega)$ and

$$
\|v\|_{2, \omega} \leq C\left(\|f\|_{\omega}+\left\|g_{1}\right\|_{H^{\frac{3}{2}\left(\partial \omega_{1}\right)}}+\left\|g_{2}\right\|_{H^{\frac{1}{2}}\left(\partial \omega_{2}\right)}\right) .
$$

Before the proof, we see that, by applying Green's formula, (1.2) is equivalent to $\left(P_{\epsilon}\right)$, which reads as:

$$
\left\{\begin{array}{l}
\text { Find } u_{\epsilon} \in H_{0}^{1}(D) \text { such that } \\
-\Delta u_{\epsilon}=f \text { in } \Omega, \\
\Delta u_{\epsilon}=0 \text { in } \Omega_{1}, \\
\left.\frac{\partial u_{\epsilon}}{\partial n}\right|_{\Gamma, \Omega}=\left.\frac{1}{\epsilon} \frac{\partial u_{\epsilon}}{\partial n}\right|_{\Gamma, \Omega_{1}},
\end{array}\right.
$$


where, for example,

$$
\left.\frac{\partial u_{\epsilon}}{\partial n}\right|_{\Gamma, \Omega}=\left.\nabla u_{\epsilon} \cdot n\right|_{\Gamma, \Omega} .
$$

From $\left(P_{\epsilon}\right)$, we see that, if we have $\left.u_{\epsilon}\right|_{\Omega} \in H^{2}(\Omega)$ with $\left\|u_{\epsilon}\right\|_{2, \Omega} \leq C$, then we have

$$
\left.\frac{\partial u_{\epsilon}}{\partial n}\right|_{\Gamma, \Omega_{1}}=\left.\epsilon \frac{\partial u_{\epsilon}}{\partial n}\right|_{\Gamma, \Omega} \in H^{\frac{1}{2}}(\Gamma) .
$$

Moreover, according to the trace theorem, we have

$$
\left\|\frac{\partial u_{\epsilon}}{\partial n}\right\|_{\frac{1}{2}, \Gamma} \leq C\left\|u_{\epsilon}\right\|_{2, \Omega} \leq C .
$$

Then, applying Lemma 3.2, we obtain

$$
\begin{aligned}
\left\|u_{\epsilon}\right\|_{2, \Omega_{1}} & \leq C\left(\|0\|_{\Omega}+\|0\|_{H^{\frac{3}{2}}(\partial D)}+\left\|\left.\frac{\partial u_{\epsilon}}{\partial n}\right|_{\Gamma, \Omega_{1}}\right\|_{\frac{1}{2}, \Gamma}\right) \\
& =C\left\|\left.\epsilon \frac{\partial u_{\epsilon}}{\partial n}\right|_{\Gamma, \Omega}\right\|_{\frac{1}{2}, \Gamma} \leq C \epsilon .
\end{aligned}
$$

Now, we can state the following proof.

Proof of Theorem 3.1 From the discussion above, we only need to show that $\left.u_{\epsilon}\right|_{\Omega} \in$ $H^{2}(\Omega)$ and $\left\|u_{\epsilon}\right\|_{2, \Omega} \leq C$. This is a well-known result; however, we want to present a brief proof here, because we will show that, by a slight change of this process, we can obtain a higher-order regularity, with smoother assumptions on $f$.

There exist $\left\{U_{j}\right\}_{j=1}^{N}, \quad U_{j} \in \mathbb{R}^{2}, \Phi_{j} \in C^{\infty}\left(\mathbb{R}^{2} ; \mathbb{R}^{2}\right)$, with $\Psi_{j}=\Phi_{j}^{-1}$, $j=1,2, \ldots, N$.

$$
\begin{gathered}
\bar{\Omega} \subset \cup_{j=1}^{N} \Phi_{j}\left(U_{j}\right) \subset D . U_{j_{0}}:=\Psi_{j}\left(\Phi_{j}\left(U_{j}\right) \cap \Omega\right)=\mathbb{R}_{+}^{2} \cap U_{j}, \\
U_{j_{1}}:=\Psi_{j}\left(\Phi_{j}\left(U_{j}\right) \cap \Omega_{1}\right)=\mathbb{R}_{-}^{2} \cap U_{j}, \quad j=1,2, \ldots, N .
\end{gathered}
$$

And also, there exists $\theta_{j} \in C_{0}^{\infty}(\bar{\Omega})$ with supp $\theta_{j} \subset \Phi_{j}\left(U_{j}\right), j=1,2, \ldots, N$, with

$$
\sum_{j=1}^{N} \theta_{j}=1, \text { on } \bar{\Omega} .
$$

Hence, $\left(\theta_{j} u_{\epsilon}\right) \circ \Phi_{j} \in H_{0}^{1}\left(U_{j}\right), j=1,2, \ldots, N$. Now we write $U, U_{1}, U_{0}, \Phi, \Psi, \theta$ instead of $U_{j}, U_{j_{1}}, U_{j_{0}}, \Phi_{j}, \Psi, \theta_{j}$. Setting $u_{2}:=\left(\theta_{j} u_{\epsilon}\right) \circ \Phi_{j}$, we investigate $u_{2}$ in the $H^{2}$ space for two cases. 
(1) The case $U_{1}=\emptyset$. We find $u_{1}:=\theta u_{\epsilon}$ satisfies

$$
\left(\nabla u_{1}, \nabla v\right)_{\Omega}=\left(\theta f-\nabla u_{\epsilon} \nabla \theta-\nabla\left(u_{\epsilon} \nabla \theta\right), v\right), \forall v \in H_{0}^{1}(\Omega)
$$

Since $\left.\left(\theta f-\nabla u_{\epsilon} \nabla \theta-\nabla\left(u_{\epsilon} \nabla \theta\right)\right)\right|_{\Omega} \in L^{2}(\Omega)$, obviously, we have

$$
\left.u_{1}\right|_{\Omega} \in H^{2}(\Omega),\left\|u_{1}\right\|_{2} \leq C .
$$

So, $u_{2} \in H^{2}(U)$ and $\left\|u_{2}\right\|_{2, U} \leq C$.

(2) The case $U_{0} \neq \varnothing$, and $U_{1} \neq \varnothing$. Writing $D_{i}=\frac{\partial}{\partial x_{i}}, \quad(i=1,2)$, we have $u_{2} \in H_{0}^{1}(U)$ satisfies

$$
\begin{aligned}
& \sum_{i, j=1}^{2} \int_{U_{0}} a_{i j} D_{i} u_{2} D_{j} v d x+\frac{1}{\epsilon} \sum_{i, j=1}^{2} \int_{U_{1}} a_{i j} D_{i} u_{2} D_{j} v d x \\
& -\int_{U_{0}}\left(u_{\epsilon} \nabla \theta\right) \circ \Phi \nabla v \nabla \Psi|D \Phi| d x-\frac{1}{\epsilon} \int_{U_{1}}\left(u_{\epsilon} \nabla \theta\right) \circ \Phi \nabla v \nabla \Psi|D \Phi| d x \\
= & \left(f_{1} \circ \Phi|D \Phi|, v\right), \forall v \in H_{0}^{1}(U),
\end{aligned}
$$

where

$$
a_{i j}=\left(\sum_{k=1}^{2} D_{k} \psi_{i} D_{k} \psi_{j}\right) \circ \Phi|D \Phi|, i, j=1,2, \Psi=\left(\psi_{1}, \psi_{2}\right) \text {. }
$$

Let $\tilde{u}_{2}$ be the zero extension of $u_{2}$ onto $\mathbb{R}^{2}$. Substituting

$$
v=\frac{\tau_{h}-1}{h} \frac{\tau_{-h}-1}{h} \tilde{u}_{2}
$$

into the above equation, where $\tau_{h}$ is the translation operator with $\tau_{h} \phi(x)=\phi\left(x_{1}+\right.$ $\left.h, x_{2}\right), \phi(x) \in L^{2}\left(\mathbb{R}^{2}\right)$. Using some lemmas of $\frac{\tau_{h}-1}{h}$ in Chapter 2 of [3], we can obtain

$$
\begin{aligned}
& \sum_{i=1}^{2}\left\|D_{i}\left(\frac{\tau_{h}-1}{h} \tilde{u}_{2}\right)\right\|_{U_{0}}^{2}+\frac{1}{\epsilon} \sum_{i=1}^{2}\left\|D_{i}\left(\frac{\tau_{h}-1}{h} \tilde{u}_{2}\right)\right\|_{U_{1}}^{2} \\
\leq & C \sum_{i=1}^{2}\left(\left\|D_{i}\left(\frac{\tau_{h}-1}{h} \tilde{u}_{2}\right)\right\|_{U_{0}}+\left\|D_{i}\left(\frac{\tau_{h}-1}{h} \tilde{u}_{2}\right)\right\|_{U_{1}}\right)
\end{aligned}
$$

Thus, we get

$$
\sum_{i=1}^{2}\left\|D_{i}\left(\frac{\tau_{h}-1}{h} \tilde{u}_{2}\right)\right\|_{U_{0}} \leq C
$$


For $i=1,2$, we consider any sequence $h_{j} \rightarrow 0$, as $j \rightarrow \infty$. We see that $D_{i} \frac{\tau_{h_{j}}-1}{h_{j}} \tilde{u}_{2}$ converges weakly to some function $\phi \in L^{2}\left(\mathbb{R}^{2}\right)$, and also

$$
D_{i} \frac{\tau_{h_{j}}-1}{h_{j}} \tilde{u}_{2} \rightarrow D_{i} D_{1} \tilde{u}_{2} \text {, as } j \rightarrow \infty,
$$

in the sense of distribution. Consequently, $D_{i} D_{1} \tilde{u}_{2}=\phi \in L^{2}\left(\mathbb{R}_{+}^{2}\right)$. This shows that all second derivatives, except $D_{2}^{2} u_{2}$, are in $L^{2}\left(\mathbb{R}^{2}\right)$. We find that the equation of $u_{2}$ is also equivelent to

$$
\begin{aligned}
& \left(\nabla u_{1}, \nabla v\right)_{\Omega}+\frac{1}{\epsilon}\left(\nabla u_{\epsilon}, \nabla v\right)_{\Omega_{1}} \\
= & (\theta \tilde{f}, v)-\left(\nabla u_{\epsilon}, v \nabla \theta\right)_{\Omega}-\frac{1}{\epsilon}\left(\nabla u_{\epsilon}, v \nabla \theta\right)_{\Omega_{1}}-\left(\nabla\left(u_{\epsilon} \nabla \theta\right)\right)_{\Omega}-\frac{1}{\epsilon}\left(\nabla\left(u_{\epsilon} \nabla \theta\right)\right)_{\Omega_{1}} \\
& +\left.\int_{\Gamma}\left(u_{\epsilon} \nabla \theta v\right)\right|_{\Gamma, \Omega} \cdot \mathbf{n} d \sigma-\left.\frac{1}{\epsilon} \int_{\Gamma}\left(u_{\epsilon} \nabla \theta v\right)\right|_{\Gamma, \Omega_{1}} \cdot \mathbf{n} d \sigma \\
:= & \left(f_{3}, v\right)_{D}+(g, v)_{\Gamma}, \forall v \in H_{0}^{1}(D) .
\end{aligned}
$$

As $\left\|f_{3}\right\|_{0, D} \leq C$ and $\|g\|_{\frac{1}{2}, \Gamma} \leq C\left(\right.$ since $\left.\left\|u_{\epsilon}\right\|_{1, \Omega_{1}} \leq C \epsilon\right)$, there exists $w \in H^{2}(\Omega)$ satisfying that $\frac{\partial w}{\partial n}=g$. Thus, $\left(u_{1}-w\right) \circ \Phi=u_{2}-w \circ \Phi$, and from the equation of $u_{1}$ above, $w$ satisfies

$$
\sum_{i, j=1}^{2} D_{j}\left(a_{i j}\left(D_{i}\left(u_{2}-w \circ \Phi\right)\right)\right)=\left(f_{3}+\Delta w\right) \circ \Phi \text { in } U_{0},
$$

which comes from Green's formula.

Thus we have $D_{2}^{2} u_{2} \in L^{2}\left(U_{0}\right)$, so that $\left\|u_{2}\right\|_{2, U_{0}} \leq C$. And because for every $j=1,2, \ldots, N,\left\|u_{2}\right\|_{2, U_{j_{0}}} \leq C$. We have $\left\|u_{\epsilon}\right\|_{2, \Omega} \leq C$.

Remark 7 With the assumption that $f \in H^{1}(\Omega)$. In the above proof, we find that by taking $v=\frac{\tau_{h}-1}{h} \frac{\tau_{h}-1}{h} \frac{\tau_{-h}-1}{h} \tilde{u}_{2}$ or $\frac{\tau_{h}-1}{h} \frac{\tau_{-h}-1}{h} \frac{\tau_{-h}-1}{h} \tilde{u}_{2}$ on case(2) instead of $\frac{\tau_{h}-1}{h} \frac{\tau_{-h}-1}{h} \tilde{u}_{2}$, we can obtain $D_{1}^{3} u_{2}, D_{1}^{2} D_{2} u_{2}, D_{2}^{2} D_{1} u_{2} \in L^{2}\left(U_{0}\right)$. Noticing that in this time $f_{3} \circ \Phi \in H^{1}\left(U_{0}\right), g \in H^{\frac{3}{2}}(\Gamma)$, we have $\|u\|_{3, \Omega} \leq C$. And applying the $\left(P_{\epsilon}\right)$, we have $\|u\|_{3, \Omega_{1}} \leq C \epsilon$. Hence, we can obtain higher-order regularity of $\left(Q_{\epsilon}\right)$.

By an analogue of the proof of Theorem 3.1 and the Remark 7, we have 
Theorem 3.3 Under the assumption that $f \in H^{k}(\Omega)$, for all non-negative integers $k$, we have

$$
\begin{aligned}
& \left.u_{\epsilon}\right|_{\Omega} \in H^{k+2}(\Omega),\left.u_{\epsilon}\right|_{\Omega_{1}} \in H^{k+2}\left(\Omega_{1}\right) . \\
& \left\|\left.u_{\epsilon}\right|_{\Omega}\right\|_{k+2, \Omega} \leq C\|f\|_{k, \Omega} \\
& \left\|\left.u_{\epsilon}\right|_{\Omega_{1}}\right\|_{k+2, \Omega_{1}} \leq C \epsilon\|f\|_{k, \Omega} .
\end{aligned}
$$

Remark 8 In the above two theorems, we both assume that $D$ is sufficiently smooth; however, in our case, $D$ is a rectangle (a convex polygon). From the discussion in $[3,4]$ on elliptic problems in non-smooth domains, we can keep Theorem 3.1 remains true for any convex polygon $D$.

\subsection{Neumann boundary value problem}

As a first step, let us assume $D$ is sufficiently smooth. Then, we have the following theorem.

Theorem 3.4 $(Q)$ is the original problem with homogeneous Neumann boundary. $f \in L^{2}(\Omega)$, then the corresponding $H^{1}$-penalty problem $\left(Q_{\epsilon}\right)$ has a unique solution $u_{\epsilon} \in H_{0}^{1}(D)$. Moreover,

$$
\begin{aligned}
& \left.u_{\epsilon}\right|_{\Omega} \in H^{2}(\Omega),\left.u_{\epsilon}\right|_{\Omega_{1}} \in H^{2}\left(\Omega_{1}\right), \\
& \left\|\left.u_{\epsilon}\right|_{\Omega}\right\|_{2, \Omega} \leq C\|f\|_{0, \Omega}, \\
& \left\|\left.u_{\epsilon}\right|_{\Omega_{1}}\right\|_{2, \Omega_{1}} \leq C\|f\|_{0, \Omega} .
\end{aligned}
$$

Before the proof, we show that, by applying the Green's formula, $\left(Q_{\epsilon}\right)$ is equivalent to $\left(P_{\epsilon}\right)$, which reads as:

$$
\left\{\begin{array}{l}
\text { Find } u_{\epsilon} \in H_{0}^{1}(D) \text { such that } \\
-\Delta u_{\epsilon}+u_{\epsilon}=f \text { in } \Omega \\
\Delta u_{\epsilon}=0 \text { in } \Omega_{1}, \\
\left.\frac{\partial u_{\epsilon}}{\partial n}\right|_{\Gamma, \Omega}=\left.\epsilon \frac{\partial u_{\epsilon}}{\partial n}\right|_{\Gamma, \Omega_{1}} .
\end{array}\right.
$$

From $\left(P_{\epsilon}\right)$ we know that, if we have $\left.u_{\epsilon}\right|_{\Omega} \in H^{2}(\Omega)$ with $\left\|u_{\epsilon}\right\|_{2, \Omega} \leq C$, then we have

$$
\left.u_{\epsilon}\right|_{\Gamma, \Omega_{1}}=\left.u_{\epsilon}\right|_{\Gamma, \Omega} \in H^{\frac{3}{2}}(\Omega)
$$

Since the right-hand-side function is 0 , and with the homogeneous Dirichlet boundary of $D$, it concludes that $\left.u_{\epsilon}\right|_{\Omega_{1}} \in H^{2}\left(\Omega_{1}\right)$ and

$$
\left\|u_{\epsilon}\right\|_{2, \Omega_{1}} \leq C\left\|u_{\epsilon}\right\|_{\frac{3}{2}, \Gamma} \leq C
$$

This means we have left to prove only $\left.u_{\epsilon}\right|_{\Omega} \in H^{2}(\Omega)$. 
Proof The process of the proof is very similar to that of Theorem 3.1. In fact, we only need to replace the $\frac{1}{\epsilon}$ in the proof of Theorem 3.1 by $\epsilon$.

Remark 9 The same comments as those in the Dirichlet case apply here, specifically, we can obtain higher-order regularity for $f \in H^{k}(\Omega)$, and, if $D$ is a convex polygon, Theorem 3.4 remains true.

\subsection{Mixed boundary value problem}

The $\left(Q_{\epsilon}\right)$ for original problem with homogeneous mixed boundary is equivalent to the problem $\left(P_{\epsilon}\right)$ :

$$
\left\{\begin{array}{l}
\text { Find } u_{\epsilon} \in H_{0}^{1}(D) \text { such that } \\
-\Delta u_{\epsilon}=f \text { in } \Omega, \\
\Delta u_{\epsilon}=0 \text { in } \Omega_{1}, \\
\Delta u_{\epsilon}=0 \text { in } \Omega_{2}, \\
\left.\frac{\partial u_{\epsilon}}{\partial n}\right|_{\Gamma_{1}, \Omega}=\left.\frac{1}{\epsilon} \frac{\partial u_{\epsilon}}{\partial n}\right|_{\Gamma_{1}, \Omega_{1}}, \\
\left.\frac{\partial u_{\epsilon}}{\partial n}\right|_{\Gamma_{2}, \Omega}=\left.\epsilon \frac{\partial u_{\epsilon}}{\partial n}\right|_{\Gamma_{2}, \Omega_{2}}, \\
\left.\frac{1}{\epsilon} \frac{\partial u_{\epsilon}}{\partial n}\right|_{\Gamma_{3}, \Omega_{1}}=\left.\epsilon \frac{\partial u_{\epsilon}}{\partial n}\right|_{\Gamma_{3}, \Omega_{2}},
\end{array},\right.
$$

where $\Gamma_{3}$ is the common boundary of $\Omega_{1}$ and $\Omega_{2}$. By an analogue of the previous proof, we can obtain that $\left.u_{\epsilon}\right|_{\Omega} \in H^{2}(\Omega)$.

Since the domain $\Omega_{1}$ and $\Omega_{2}$ have corners at the intersection points of their boundaries (see Fig. 1), $\left.u_{\epsilon}\right|_{\Omega_{1}}$ and $\left.u_{\epsilon}\right|_{\Omega_{2}}$ would not be in $H^{2}$ space but $H^{1+\alpha}, \alpha \in(0,1)$ (see $[4,9])$.

\section{Finite element approximation and discrete problems}

Recall that the Cartesian mesh is introduced to the rectangular domain $D$ to get a uniform triangulation $\mathcal{T}_{h}$, and $h$ is the maximum diameter of the triangles of $\mathcal{T}_{h}$. Each $K \in \mathcal{T}_{h}$ is assumed to be a closed set. $V_{h}(D) \subset H_{0}^{1}(D)$ is the subspace of all piecewise linear continuous functions subordinate to $\mathcal{T}_{h}$.

\subsection{Dirichlet boundary value problem}

We consider the discrete problem (1.3).

Lemma 4.1 There exists a unique solution $u_{\epsilon, h} \in V_{h}(D)$ for (1.3). $u_{\epsilon}$ is the solution of (1.2), and we have

$$
\begin{aligned}
& \left\|u_{\epsilon}-u_{\epsilon, h}\right\|_{1, \Omega}+\frac{1}{\sqrt{\epsilon}}\left\|u_{\epsilon}-u_{\epsilon, h}\right\|_{1, \Omega_{1}} \\
\leq & C \inf _{v_{h} \in V_{h}(D)}\left(\left\|u_{\epsilon}-v_{h}\right\|_{1, \Omega}+\frac{1}{\sqrt{\epsilon}}\left\|u_{\epsilon}-v_{h}\right\|_{1, \Omega_{1}}\right) .
\end{aligned}
$$


Proof Subtracting (1.2) from (1.3), we have

$$
\left(\nabla\left(u_{\epsilon}-u_{\epsilon, h}\right), \nabla v_{h}\right)_{\Omega}+\frac{1}{\epsilon}\left(\nabla\left(u_{\epsilon}-u_{\epsilon, h}\right), \nabla v_{h}\right)_{\Omega_{1}}=0, \forall v_{h} \in V_{h}(D) .
$$

Then we find

$$
\begin{aligned}
& \left|u_{\epsilon}-u_{\epsilon, h}\right|_{1, \Omega}^{2}+\frac{1}{\epsilon}\left|u_{\epsilon}-u_{\epsilon, h}\right|_{1, \Omega_{1}}^{2} \\
\leq & \inf _{v_{h} \in V_{h}(D)}\left\{\left(\nabla\left(u_{\epsilon}-u_{\epsilon, h}\right), \nabla\left(u_{\epsilon}-v_{h}\right)\right)_{\Omega}+\frac{1}{\epsilon}\left(\nabla\left(u_{\epsilon}-u_{\epsilon, h}\right), \nabla\left(u_{\epsilon}-v_{h}\right)\right)_{\Omega_{1}}\right\} \\
\leq & \inf _{v_{h} \in V_{h}(D)}\left\{\left|u_{\epsilon}-u_{\epsilon, h}\right|_{1, \Omega}\left|u_{\epsilon}-v_{h}\right|_{1, \Omega}+\frac{1}{\epsilon}\left|u_{\epsilon}-u_{\epsilon, h}\right|_{1, \Omega_{1}}\left|u_{\epsilon}-v_{h}\right|_{1, \Omega_{1}}\right\} \\
\leq & \left\{\left\|u_{\epsilon}-u_{\epsilon, h}\right\|_{1, \Omega}+\frac{1}{\sqrt{\epsilon}}\left\|u_{\epsilon}-u_{\epsilon, h}\right\|_{1, \Omega_{1}}\right\} \\
& \times \inf _{v_{h} \in V_{h}(D)}\left\{\left\|u_{\epsilon}-v_{h}\right\|_{1, \Omega}+\frac{1}{\sqrt{\epsilon}}\left\|u_{\epsilon}-v_{h}\right\|_{1, \Omega_{1}}\right\} .
\end{aligned}
$$

Applying the Poincaré inequality to the left-hand-side, we have

$$
\begin{aligned}
& \left|u_{\epsilon}-u_{\epsilon, h}\right|_{1, \Omega}^{2}+\frac{1}{\epsilon}\left|u_{\epsilon}-u_{\epsilon, h}\right|_{1, \Omega_{1}}^{2} \\
= & \left|u_{\epsilon}-u_{\epsilon, h}\right|_{1, D}^{2}+\left(\frac{1}{\epsilon}-1\right)\left|u_{\epsilon}-u_{\epsilon, h}\right|_{1, \Omega_{1}}^{2} \\
\geq & C\left(\left\|u_{\epsilon}-u_{\epsilon, h}\right\|_{1, \Omega}^{2}+\frac{1}{\sqrt{\epsilon}}\left\|u_{\epsilon}-u_{\epsilon, h}\right\|_{1, \Omega_{1}}^{2}\right)
\end{aligned}
$$

Thus, we have proved the result.

To estimate

$$
\inf _{v_{h} \in V_{h}(D)}\left(\left\|u_{\epsilon}-v_{h}\right\|_{1, \Omega}+\frac{1}{\sqrt{\epsilon}}\left\|u_{\epsilon}-v_{h}\right\|_{1, \Omega_{1}}\right),
$$

we need some lemmas, which can be found in [12], and several other similar results in $[10,13]$. For a curve $\gamma$ in $C^{2}\left(\mathbb{R}^{2}\right)$ and $\delta>0$, we define a $\delta$-neighborhood $\gamma_{\delta}=\{x \in$ $\left.\mathbb{R}^{2} \mid \operatorname{dist}(x, \gamma) \leq \delta\right\}$

Lemma 4.2 Suppose $\gamma_{\delta} \subset \subset R, R$ is a domain in $\mathbb{R}^{2}$, and $v \in H^{1}(R)$. Then we have

$$
\|v\|_{0, \gamma_{\delta}} \leq C \sqrt{\delta}\|v\|_{1, R}
$$

If we assume $v \in H^{2}(R)$, then we have

$$
\|v\|_{1, \gamma_{\delta}} \leq C \sqrt{\delta}\|v\|_{2, R}
$$


Lemma 4.3 Suppose $w \in H^{2}(D)$, and we define $I_{K} w$ as the linear interpolation of $w$ on the vertices of a triangle $K \in \mathcal{T}_{h}$. Then, we have

$$
\left|I_{K} w\right|_{1, K} \simeq \sum_{i, j=1, i \neq j}^{3}\left|w\left(v_{i}\right)-w\left(v_{j}\right)\right|
$$

where $v_{i}, i=1,2,3$, are vertices of $K .(A \simeq B$ means that there exist constants depending on the regularity of the triangulation $C_{1}, C_{2}$ such that $C_{2} B \leq A \leq C_{1} B$.)

Theorem 4.4 The following error estimate for (1.2) and (1.3) holds:

$$
\left\|u_{\epsilon}-u_{\epsilon, h}\right\|_{1, \Omega}+\frac{1}{\sqrt{\epsilon}}\left\|u_{\epsilon}-u_{\epsilon, h}\right\|_{1, \Omega_{1}} \leq C(\sqrt{h}+\sqrt{\epsilon})\left(\left\|u_{\epsilon}\right\|_{2, \Omega}+\left\|u_{\epsilon}\right\|_{2, \Omega_{1}}\right) .
$$

Before the proof, we define some notations:

$$
\begin{aligned}
& \Lambda_{h}=\text { the set of all vertices of } \mathcal{T}_{h}, \\
& \Lambda(K)=\left\{v_{1}^{K}, v_{2}^{K}, v_{3}^{K}\right\}=\text { the set of all vertices of } K \in \mathcal{T}_{h}, \\
& T_{\Omega}=\left\{K \in \mathcal{T}_{h} \mid K \subset \Omega, K \cap \Gamma=\emptyset\right\}, \\
& T_{\Omega_{1}}=\left\{K \in \mathcal{T}_{h} \mid K \subset \Omega_{1}, K \cap \Gamma=\emptyset\right\}, \\
& T_{\Gamma}=\left\{K \in \mathcal{T}_{h} \mid K \cap \Gamma \neq \emptyset\right\}, \\
& T_{0}=\left\{K \in T_{\Omega} \mid K \cap T_{\Gamma} \neq \emptyset\right\}, \\
& T_{1}=\left\{K \in T_{\Omega_{1}} \mid K \cap T_{\Gamma} \neq \emptyset\right\}, \\
& \overline{\omega_{0}}=\cup_{K \in T_{0}} K, \\
& \overline{\omega_{\Omega}}=\cup_{K \in T_{\Omega} \backslash T_{0}} K, \\
& \overline{\omega_{\Gamma}}=\cup_{K \in T_{\Gamma}} K .
\end{aligned}
$$

We may assume that $T_{\Omega} \backslash T_{0} \neq \varnothing$ and $T_{\Omega_{1}} \backslash T_{1} \neq \varnothing$ without loss of generality.

Proof of Theorem 4.4 We define $v_{h}$ by setting,

$$
v_{h}(v)= \begin{cases}u_{\epsilon}(v) & \text { for } v \in \Lambda(K), K \in T_{\Omega} \backslash T_{0}, \\ 0 & \text { for all others vertices } v\end{cases}
$$

and substitute this $v_{h}$ into the right-hand-side of (4.1). We find that $\left\|u_{\epsilon}-v_{h}\right\|_{1, \Omega_{1}}=$ $\left\|u_{\epsilon}\right\|_{1, \Omega_{1}} \leq C \epsilon$. To estimate $\left\|u_{\epsilon}-v_{h}\right\|_{1, \Omega}$, we use the scheme proposed in [12] by using Lemmas 4.2 and 4.3. However, there are several differences between our analysis and that of [12], because we apply our regularity theorem presented in the previous section, which simplifies the analysis.

We find that $\left\|u_{\epsilon}-v_{h}\right\|_{1, \Omega}^{2}=\left\|u_{\epsilon}-v_{h}\right\|_{1, \omega_{\Omega}}^{2}+\left\|u_{\epsilon}-v_{h}\right\|_{1, \omega_{0}}^{2}+\left\|u_{\epsilon}-v_{h}\right\|_{1, \omega_{\Gamma}}^{2}$.

For the first term $\left\|u_{\epsilon}-v_{h}\right\|_{1, \omega_{\Omega}}=\left\|u_{\epsilon}-I_{K} u_{\epsilon}\right\|_{1, \omega_{\Omega}} \leq C h\left\|u_{\epsilon}\right\|_{2, \Omega}$.

For the third term $\left\|u_{\epsilon}-v_{h}\right\|_{1, \omega_{\Gamma}}=\left\|u_{\epsilon}-0\right\|_{1, T_{\omega_{\Gamma}}} \leq C \sqrt{h}\left\|u_{\epsilon}\right\|_{2, \Omega}$, following from Lemma 4.2. 
Then, for the second term $\left\|u_{\epsilon}-v_{h}\right\|_{1, \omega_{0}} \leq\left\|u_{\epsilon}\right\|_{1, \omega_{0}}+\left\|v_{h}\right\|_{1, \omega_{0}}$, and we have $\left\|u_{\epsilon}\right\|_{1, \omega_{0}} \leq C \sqrt{h}\left\|u_{\epsilon}\right\|_{2, \Omega}$, again, following from Lemma 4.2. What remains is to estimate $\left\|v_{h}\right\|_{1, \omega_{0}}$.

For every $K \in T_{0}$, we have

$$
\begin{aligned}
& \left\|v_{h}\right\|_{1, K} \leq\left\|v_{h}-I_{K} u_{\epsilon}\right\|_{1, K}+\left\|I_{K} u_{\epsilon}\right\|_{1, K} \\
\leq & \left|v_{h}-I_{K} u_{\epsilon}\right|_{1, K}+\left\|v_{h}-I_{K} u_{\epsilon}\right\|_{0, K}+\left\|I_{K} u_{\epsilon}\right\|_{1, K} \\
\leq & \left|v_{h}-I_{K} u_{\epsilon}\right|_{1, K}+\left\|v_{h}\right\|_{0, K}+\left\|I_{K} u_{\epsilon}\right\|_{0, K}+\left\|I_{K} u_{\epsilon}\right\|_{1, K} .
\end{aligned}
$$

We want to show that $\left\|v_{h}\right\|_{0, K} \leq C\left\|I_{K} u_{\epsilon}\right\|_{1, K}$. There are two possibilities: (1) if for all $v_{i}=v_{i}^{K} \in \Lambda(K), i=1,2,3, u_{\epsilon}\left(v_{i}\right)$ have the same sign, then obviously, $\left|v_{h}(x)\right|^{2} \leq\left|I_{K} u_{\epsilon}(x)\right|^{2}, \quad(x \in K)$ and $\left\|v_{h}\right\|_{0, K} \leq C\left\|I_{K} u_{\epsilon}\right\|_{0, K}$; (2) if we consider the case where for $v_{i}, i=1,2,3, u_{i}=u_{\epsilon}\left(v_{i}\right)$ do not all have the same signs, then, without loss of generality, we assume that $\left|u_{1}\right|=\|u\|_{\infty, K}$ and $u_{1} u_{2} \geq 0$ and $u_{3} \leq 0$. We have $\nabla\left(I_{K} u\right) \cdot \overrightarrow{v_{3} v_{1}}=u_{1}-u_{3}$ on $K$. Hence, $\left|u_{1}\right| \leq\left|u_{1}-u_{3}\right| \leq\left|\nabla\left(I_{K} u\right)\right| \cdot\left|\overrightarrow{v_{3} v_{1}}\right| \leq$ $\left|\nabla\left(I_{K} u\right)\right| h_{K}$ on $K$, since $u_{1}$ and $u_{3}$ have the different signs. Therefore,

$$
\begin{aligned}
\left\|v_{h}\right\|_{0, K} & \leq|K|^{\frac{1}{2}}\left\|v_{h}\right\|_{\infty, K} \\
& \leq|K|^{\frac{1}{2}}\left\|u_{\epsilon}\right\|_{\infty, K} \\
& \leq|K|^{\frac{1}{2}}\left|\nabla I_{K} u\right| h_{K}=h_{K}\left|\nabla\left(I_{K} u\right)\right|_{0, K} .
\end{aligned}
$$

Thus, we have $\left\|v_{h}\right\|_{0, K} \leq\left(1+h_{K}\right)\left\|I_{K} u\right\|_{1, K}$, which gives

$$
\begin{aligned}
\left\|v_{h}\right\|_{1, K} & \leq\left|v_{h}-I_{K} u_{\epsilon}\right|_{1, K}+C\left\|I_{K} u_{\epsilon}\right\|_{1, K} \\
& \leq\left|v_{h}-I_{K} u_{\epsilon}\right|_{1, K}+C\left\|I_{K} u_{\epsilon}-u_{\epsilon}\right\|_{1, K}+C\left\|u_{\epsilon}\right\|_{1, K}
\end{aligned}
$$

By the standard interpolation error estimates, we have

$$
\sum_{K \in T_{0}}\left\|I_{K} u_{\epsilon}-u_{\epsilon}\right\|_{1, K}^{2} \leq C h^{2}\left\|u_{\epsilon}\right\|_{2, \Omega}^{2} .
$$

We notice that there exists $C^{\prime}>0$ such that $\overline{\omega_{\Gamma}} \subset \gamma_{C^{\prime} h}$. By Lemma 4.3, setting $\hat{v}_{h}=v_{h}-I_{K} u_{\epsilon}$, we obtain

$$
\left|v_{h}-I_{K} u_{\epsilon}\right|_{1, K} \leq C \sum_{\substack{v_{i}, v_{j} \in \Lambda(K) \\ i \neq j}}\left|\hat{v}_{h}\left(v_{i}\right)-\hat{v}_{h}\left(v_{j}\right)\right| .
$$

Next, we set

$$
\begin{aligned}
& \Lambda_{\Omega}(K)=\left\{v \in \Lambda(K) \mid \exists K^{\prime} \in T_{\Omega} \backslash T_{0}, \text { s.t. } v \text { in } \Lambda\left(K^{\prime}\right)\right\}, \\
& \Lambda_{\Gamma}(K)=\left\{v \in \Lambda(K) \mid \exists K^{\prime} \in T_{\Gamma}, \text { s.t. } v \text { in } \Lambda\left(K^{\prime \prime}\right)\right\} .
\end{aligned}
$$


By definition, we see that $\Lambda(K)=\Lambda_{\Omega}(K) \cup \Lambda_{\Gamma}(K), \Lambda_{\Omega}(K) \neq \emptyset, \Lambda_{\Gamma}(K) \neq \emptyset$. There are two possibilities: for $\left\{v_{i}=v_{i}^{K}\right\}_{i=1}^{3}=\Lambda(K)$,

(i) $\Lambda_{\Omega}(K)=\left\{v_{1}, v_{2}\right\}, \Lambda_{\Gamma}(K)=\left\{v_{3}\right\}$;

(ii) $\Lambda_{\Omega}(K)=\left\{v_{1}\right\}, \Lambda_{\Gamma}(K)=\left\{v_{2}, v_{3}\right\}$.

(See Figs. 2 and 3.)

For (i), we have

$$
\begin{aligned}
& \hat{v}_{h}\left(v_{i}\right)=v_{h}\left(v_{i}\right)-\left(I_{K} u_{\epsilon}\right)\left(v_{i}\right)=0, i=1,2, \\
& \hat{v}_{h}\left(v_{3}\right)=v_{h}\left(v_{3}\right)-\left(I_{K} u_{\epsilon}\right)\left(v_{3}\right)=-u_{\epsilon}\left(v_{3}\right) .
\end{aligned}
$$

Fig. $2 \Lambda_{\Omega}(K)=$

$\left\{v_{1}, v_{2}\right\}, \Lambda_{\Gamma}(K)=\left\{v_{3}\right\}$

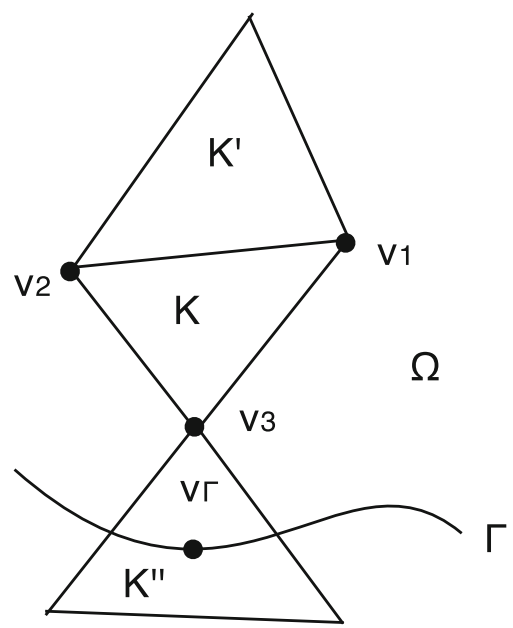

$\Omega 1$

Fig. $3 \Lambda_{\Omega}(K)=$

$\left\{v_{1}\right\}, \Lambda_{\Gamma}(K)=\left\{v_{2}, v_{3}\right\}$

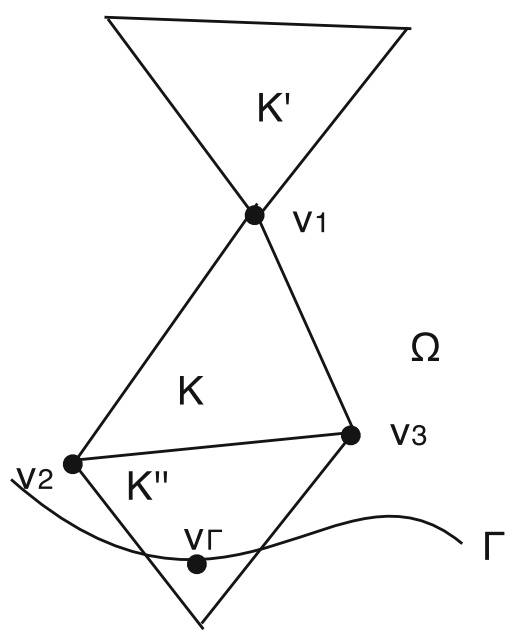

$\Omega 1$ 
Hence, $\left|\hat{v}_{h}\right|_{1, K} \leq C\left|u_{\epsilon}\left(v_{3}\right)\right|$. Taking arbitrary point $v_{\Gamma} \in \Gamma \cap K^{\prime \prime}$, we have $\left|\hat{v}_{h}\right|_{1, K} \leq$ $C\left(\left|u_{\epsilon}\left(v_{3}\right)-u_{\epsilon}\left(v_{\Gamma}\right)\right|+\left|u_{\epsilon}\left(v_{\Gamma}\right)\right|\right)$.

At this stage, we apply the Sobolev and Morrey's inequalities. Let $\omega$ be a Lipschitz domain in $\mathbb{R}^{2}$. They are given as

$$
\begin{aligned}
& \|u\|_{L^{\infty}(\omega)} \leq C\|u\|_{H^{2}(\omega)}, \quad\left(\forall u \in H^{2}(\omega)\right) \\
& \|u\|_{W^{1, q}(\omega)} \leq C\|u\|_{H^{2}(\omega)}, \quad\left(1 \leq q<\infty, \forall u \in H^{2}(\omega)\right) \\
& |u(x)-u(y)| \leq C\|u\|_{W^{1, q}(\omega)}|x-y|^{\alpha}, \\
& \left(x, y \in \omega, 2<q, \alpha=1-\frac{2}{q}, u \in W^{1, q}(\omega)\right)
\end{aligned}
$$

We choose $q=4$, and define $K_{\Omega}^{\prime \prime}=K^{\prime \prime} \cap \Omega, K_{\Omega_{1}}^{\prime \prime}=K^{\prime \prime} \cap \Omega_{1}$, and we have

$$
\begin{aligned}
& \left|u_{\epsilon}\left(v_{3}\right)-u_{\epsilon}\left(v_{\Gamma}\right)\right| \leq C\left\|u_{\epsilon}\right\|_{W^{1,4}\left(K_{\Omega}^{\prime \prime}\right)}\left|v_{3}-v_{\Gamma}\right|^{\frac{1}{2}} \leq C h^{\frac{1}{2}}\left\|u_{\epsilon}\right\|_{2, K_{\Omega}^{\prime \prime}}, \\
& \left|u_{\epsilon}\left(v_{\Gamma}\right)\right| \leq C\left\|u_{\epsilon}\right\|_{2, K_{\Omega_{1}}^{\prime \prime}} .
\end{aligned}
$$

Hence, we obtain $\left|\hat{v}_{h}\right|_{1, K} \leq C\left\|u_{\epsilon}\right\|_{2, K_{\Omega_{1}}^{\prime \prime}}+C h^{\frac{1}{2}}\left\|u_{\epsilon}\right\|_{2, K_{\Omega}^{\prime \prime}}$.

Then, applying the same trick to (ii), we can show that

$$
\begin{aligned}
\hat{v}_{h}\left(v_{1}\right) & =v_{h}\left(v_{1}\right)-\left(I_{K} u_{\epsilon}\right)\left(v_{1}\right)=0, \\
\hat{v}_{h}\left(v_{i}\right) & =v_{h}\left(v_{i}\right)-\left(I_{K} u_{\epsilon}\right)\left(v_{i}\right)=-u_{\epsilon}\left(v_{i}\right), i=2,3, \\
\left|\hat{v}_{h}\right|_{1, K} & \leq C\left(\left|u_{\epsilon}\left(v_{2}\right)-u_{\epsilon}\left(v_{3}\right)\right|+\left|u_{\epsilon}\left(v_{2}\right)\right|+\left|u_{\epsilon}\left(v_{3}\right)\right|\right) \\
& \leq C \sum_{i=2,3}\left|u_{\epsilon}\left(v_{i}\right)-u_{\epsilon}\left(v_{\Gamma}\right)\right|+C\left|u_{\epsilon}\left(v_{\Gamma}\right)\right| \\
& \leq C\left\|u_{\epsilon}\right\|_{2, K_{\Omega_{1}}^{\prime \prime}}+C h^{\frac{1}{2}}\left\|u_{\epsilon}\right\|_{2, K_{\Omega}^{\prime \prime}} .
\end{aligned}
$$

Combining (i) and (ii), we have

$$
\begin{aligned}
\sum_{K \in T_{0}}\left|\hat{v}_{h}\right|_{1, K}^{2} & \leq C \sum_{K \in T_{0}}\left(\left\|u_{\epsilon}\right\|_{2, K_{\Omega_{1}}^{\prime \prime}}^{2}+C h\left\|u_{\epsilon}\right\|_{2, K_{\Omega}^{\prime \prime}}^{2}\right) \\
& \leq C\left\|u_{\epsilon}\right\|_{2, \Omega_{1}}^{2}+C h\left\|u_{\epsilon}\right\|_{2, \Omega}^{2} .
\end{aligned}
$$

From (4.6), (4.7), (4.8), (4.9), we can easily derive that

$$
\begin{aligned}
\left\|v_{h}\right\|_{1, \omega_{0}}^{2} & =\sum_{K \in T_{0}}\left\|v_{h}\right\|_{1, K}^{2} \\
& \leq\left(\left\|u_{\epsilon}\right\|_{2, \Omega_{1}}^{2}+h\left\|u_{\epsilon}\right\|_{2, \Omega}^{2} h^{2}\left\|u_{\epsilon}\right\|_{2, \Omega}^{2}+h\left\|u_{\epsilon}\right\|_{2, \Omega}^{2}\right) \\
& \leq \epsilon^{2}\|f\|_{0, \Omega}^{2}+C\left(h^{2}+h\right)\|f\|_{0, \Omega}^{2},
\end{aligned}
$$


where the last inequality is from Theorem 3.1. Hence, we get

$$
\left\|v_{h}\right\|_{1, \omega_{0}} \leq C\left(\epsilon+h+h^{\frac{1}{2}}\right)\|f\|_{0, \Omega} .
$$

Recalling that $\left\|u_{\epsilon}-v_{h}\right\|_{1, \Omega_{1}}=\left\|u_{\epsilon}\right\|_{1, \Omega_{1}} \leq C \epsilon$ and other estimates from the beginning of the proof, we have

$$
\left\|u_{\epsilon}-v_{h}\right\|_{1, \Omega}+\frac{1}{\sqrt{\epsilon}}\left\|u_{\epsilon}-v_{h}\right\|_{1, \Omega_{1}} \leq C(\sqrt{\epsilon}+\sqrt{h}) .
$$

Hence, the theorem follows from Lemma 4.1.

Remark 10 Since we have $\left\|u_{\epsilon}\right\|_{2, \Omega_{1}} \leq C \epsilon$, other choices for $v_{h}$ than that above can be taken, such as

$$
v_{h}=\left\{\begin{array}{l}
u_{\epsilon}(v) \text { for } v \in \Lambda(K), \quad K \in T_{\Omega} \backslash T_{0}, \\
\bar{u}_{\epsilon}(v) \text { for } v \in \Lambda(K), \quad K \in T_{\Omega_{1}} \cup T_{\Gamma},
\end{array}\right.
$$

where $\bar{u}_{\epsilon}$ is the extension of $\left.u_{\epsilon}\right|_{\Omega_{1}}$ onto $D$ with $\left\|\bar{u}_{\epsilon}\right\|_{2, D} \leq C\left\|u_{\epsilon}\right\|_{2, \Omega_{1}}$, and the estimate result still holds.

To estimate $\left\|u_{\epsilon}-u_{\epsilon, h}\right\|_{0, D}$, we need the adjoint boundary value problem, which reads as:

$$
\left\{\begin{array}{l}
\text { For any given } f \in L^{2}(\Omega), \text { find } u_{\epsilon f} \in H_{0}^{1}(D) \text { such that } \\
\left(\nabla v, \nabla u_{\epsilon f}\right)_{\Omega}+\frac{1}{\epsilon}\left(\nabla v, \nabla u_{\epsilon f}\right)_{\Omega_{1}}=(\tilde{f}, v), \forall v \in H_{0}^{1}(D) .
\end{array}\right.
$$

( $\tilde{f}$ is the zero extension of $f$.) We see that there exists a unique solution $u_{\epsilon f} \in H_{0}^{1}(D)$, with

$$
\left\|u_{\epsilon f}\right\|_{2, \Omega} \leq C\|f\|_{0, \Omega},\left\|u_{\epsilon f}\right\|_{2, \Omega_{1}} \leq C \epsilon\|f\|_{0, \Omega},
$$

which follows from Theorem 3.1.

Theorem 4.5 We have the error estimate in $L^{2}$ norm for (1.2) and (1.3),

$$
\left\|u_{\epsilon}-u_{\epsilon, h}\right\|_{0, D} \leq C(\epsilon+h+\sqrt{h \epsilon})
$$

Proof Let $f=\left.\left(u_{\epsilon}-u_{\epsilon, h}\right)\right|_{\Omega}$ and substitute $v=u_{\epsilon}-u_{\epsilon, h}$ into the equation of the adjoint problem, we get 


$$
\begin{aligned}
& \left\|u_{\epsilon}-u_{\epsilon, h}\right\|_{0, \Omega}^{2}=\left(f, u_{\epsilon}-u_{\epsilon, h}\right)_{0, \Omega} \\
= & \left(\tilde{f}, u_{\epsilon}-u_{\epsilon, h}\right)_{0, D} \\
= & \left(\nabla\left(u_{\epsilon}-u_{\epsilon, h}\right), \nabla u_{\epsilon f}\right)_{\Omega}+\frac{1}{\epsilon}\left(\nabla\left(u_{\epsilon}-u_{\epsilon, h}\right), \nabla u_{\epsilon f}\right)_{\Omega_{1}} \\
\leq & \left(\nabla\left(u_{\epsilon}-u_{\epsilon, h}\right), \nabla\left(u_{\epsilon f}-v_{h}\right)\right)_{\Omega}+\frac{1}{\epsilon}\left(\nabla\left(u_{\epsilon}-u_{\epsilon, h}\right), \nabla\left(u_{\epsilon f}-v_{h}\right)\right)_{\Omega_{1}}, \forall v_{h} \in V_{h}(D) \\
\leq & \left\|u_{\epsilon}-u_{\epsilon, h}\right\|_{1, \Omega}\left\|u_{\epsilon f}-v_{h}\right\|_{1, \Omega}+\frac{1}{\epsilon}\left\|u_{\epsilon}-u_{\epsilon, h}\right\|_{1, \Omega_{1}}\left\|\nabla u_{\epsilon f}-v_{h}\right\|_{1, \Omega_{1}} \\
\leq & C(\sqrt{\epsilon}+\sqrt{h})(\sqrt{\epsilon}+\sqrt{h})\|f\|_{0, \Omega}+C \frac{1}{\epsilon} \sqrt{\epsilon}(\sqrt{\epsilon}+\sqrt{h}) \sqrt{\epsilon}(\sqrt{\epsilon}+\sqrt{h})\|f\|_{0, \Omega}
\end{aligned}
$$

The last inequality follows from Theorems 3.1 and 4.4. Noticing that $f=$ $\left.\left(u_{\epsilon}-u_{\epsilon, h}\right)\right|_{\Omega}$, we get

$$
\left\|u_{\epsilon}-u_{\epsilon, h}\right\|_{0, \Omega} \leq C(\sqrt{\epsilon}+\sqrt{h})^{2}
$$

With $\left\|u_{\epsilon}\right\|_{1, \Omega_{1}} \leq C \epsilon$ and $\left\|u_{\epsilon, h}\right\|_{1, \Omega_{1}} \leq C \epsilon$, we have proved the result.

4.2 Neumann boundary value problem

The discrete problem $\left(Q_{\epsilon, h}\right)$ reads as:

$$
\left\{\begin{array}{l}
\text { Find } u_{\epsilon, h} \in V_{h}(D) \text { such that } \\
\left(u_{\epsilon, h}, v_{h}\right)_{1, \Omega}+\epsilon\left(\nabla u_{\epsilon, h}, \nabla v_{h}\right)_{\Omega_{1}}=\left(\tilde{f}, v_{h}\right)_{D}, \quad \forall v_{h} \in V_{h}(D) .
\end{array}\right.
$$

Lemma 4.6 There exists a unique solution $u_{\epsilon, h} \in V_{h}(D)$ for (4.12). $u_{\epsilon}$ is the solution of (2.4), and we have

$$
\begin{aligned}
& \left\|u_{\epsilon}-u_{\epsilon, h}\right\|_{1, \Omega}+\sqrt{\epsilon}\left\|u_{\epsilon}-u_{\epsilon, h}\right\|_{1, \Omega_{1}} \\
\leq & C \inf _{v_{h} \in V_{h}(D)}\left(\left\|u_{\epsilon}-v_{h}\right\|_{1, \Omega}+\sqrt{\epsilon}\left\|u_{\epsilon}-v_{h}\right\|_{1, \Omega_{1}}\right) .
\end{aligned}
$$

Proof The proof of this lemma is an analogue of that of Lemma 4.1.

Then, we have the error estimate theorem:

Theorem 4.7 We have the error estimate for (2.4) and (4.12)

$$
\left\|u_{\epsilon}-u_{\epsilon, h}\right\|_{1, \Omega}+\sqrt{\epsilon}\left\|u_{\epsilon}-u_{\epsilon, h}\right\|_{1, \Omega_{1}} \leq C(h+\epsilon+\sqrt{h \epsilon})\left(\left\|u_{\epsilon}\right\|_{2, \Omega}+\left\|u_{\epsilon}\right\|_{2, \Omega_{1}}\right) .
$$

Proof By taking

$$
v_{h}= \begin{cases}u_{\epsilon}(v) \text { for } v \in \Lambda(K), & K \in T_{\Omega} \cup T_{\Gamma}, \\ \bar{u}_{\epsilon}(v) \text { for } v \in \Lambda(K), \quad K \in T_{\Omega_{1}} \backslash T_{1},\end{cases}
$$

the proof is an analogue of that of Theorem 4.4. 
With the analogue of the proof of the Dirichlet case, we have the error estimate in $L^{2}$ norm for Neumann case.

Theorem 4.8 For $u_{\epsilon}$ and $u_{\epsilon, h}$ are the solutions of (2.4) and (4.12), respectively, we have

$$
\left\|u_{\epsilon}-u_{\epsilon, h}\right\|_{0, D} \leq C(\epsilon+h+\sqrt{h \epsilon})^{2} .
$$

\subsection{Mixed boundary value problem}

Since the regularity theorem of $\left(Q_{\epsilon}\right)$ for mixed boundary case is weak, we will not put a discussion on the error estimates of the discrete problem for this case. Also, we could not find any discussion on this issue in $[8,12]$ etc.

\section{An approximation for discrete problems}

In the discrete problem, we find the inner-product $\left(\nabla u_{\epsilon, h}, \nabla v_{h}\right)_{\Omega}$ or $\Omega_{1}$ and $\left(\tilde{f}, v_{h}\right)_{D}$ (since $\tilde{f}$ is the zero extension of $f$ from $\Omega$ onto $D$ ) are not applicable to computation, because we assumed that $\Omega$ has a curved boundary $\Gamma$. The integral of the elements crossing $\Gamma$ becomes a problem when doing computation. Thus, we need a proper approximation. One way is to replace the integral in the open triangle $K, K \cap \Gamma \neq \emptyset$, of

$$
\left(\nabla u_{\epsilon}, v_{h}\right)_{K \cap \Omega}+\frac{1}{\epsilon}\left(\nabla u_{\epsilon}, v_{h}\right)_{K \cap \Omega_{1}}
$$

by the integral of

$$
\left(\nabla u_{\epsilon}, v_{h}\right)_{K \cap \hat{\Omega}}+\frac{1}{\epsilon}\left(\nabla u_{\epsilon}, v_{h}\right)_{K \cap \hat{\Omega_{1}}}
$$

where $\hat{\Omega}$ is a polygon with vertices which are the points of intersection between $\Gamma$ and the triangles' edges. $\hat{\Omega}$ satisfies $(\Omega \backslash \hat{\Omega}) \cup(\hat{\Omega} \backslash \Omega) \subset \Gamma_{\delta}, \delta=O\left(h^{2}\right) . \hat{\Omega}_{1}=D \backslash \overline{\hat{\Omega}}$. The approximation problem of $\left(Q_{\epsilon, h}\right)$ is denoted as $\left(\hat{Q}_{\epsilon, h}\right)$.

\subsection{Dirichlet boundary value problem}

The problem (1.4) is considered. We assume that $f_{h}$ is some interpolation of $\tilde{f}$, such that $\left(f_{h}, v_{h}\right)_{D}$ is applicable to computation and has $\left\|f_{h}-\tilde{f}\right\|_{0} \leq C h$ holds. For example, suppose $f \in C^{1}(\Omega)$; then we can choose $f_{h}$ is the linear interpolation of $f$ on the vertices $v$ of triangles for every $v \in \hat{\Omega}$ and zero on other vertices. Before giving the estimate of $\left\|\hat{u}_{\epsilon, h}-u_{\epsilon, h}\right\|_{1, D}$, we quote a lemma from [13]. For any open triangle $K$, we denote

$$
\pi_{K}=\left(\Omega_{1} \backslash \hat{\Omega}_{1}\right) \cap K=(\hat{\Omega} \backslash \Omega) \cap K
$$


and

$$
\begin{aligned}
\hat{\pi}_{K} & :=\left(\hat{\Omega}_{1} \backslash \Omega_{1}\right) \cap K=(\Omega \backslash \hat{\Omega}) \cap K . \\
\pi & :=\cup_{K} \pi_{K} . \\
\hat{\pi} & :=\cup_{K} \hat{\pi}_{K} .
\end{aligned}
$$

Lemma 5.1 As we have $(\Omega \backslash \hat{\Omega}) \cup(\hat{\Omega} \backslash \Omega) \subset \Gamma_{\delta}, \delta=O\left(h^{2}\right)$, the following estimates hold for any $v_{h} \in V_{h}$,

$$
\begin{aligned}
& \left|v_{h}\right|_{1, \pi} \leq h^{\frac{1}{2}}\left|v_{h}\right|_{1, \Omega_{1}}\left(\operatorname{or} h^{\frac{1}{2}}\left|v_{h}\right|_{1, \hat{\Omega}}\right), \\
& \left|v_{h}\right|_{1, \hat{\pi}} \leq h^{\frac{1}{2}}\left|v_{h}\right|_{1, \hat{\Omega}_{1}}\left(\operatorname{or} h^{\frac{1}{2}}\left|v_{h}\right|_{1, \Omega}\right) .
\end{aligned}
$$

Then we have the following theorem.

Theorem 5.2 There exists a unique solution $\hat{u}_{\epsilon, h}$ for (1.4). $u_{\epsilon, h}$ is the solution of (1.3), and we have

$$
\left\|u_{\epsilon, h}-\hat{u}_{\epsilon, h}\right\|_{1, D} \leq C h .
$$

Proof Subtracting the equation in (1.4) from that in (1.3), we have

$$
\begin{aligned}
& \left(\nabla\left(u_{\epsilon, h}-\hat{u}_{\epsilon, h}\right), \nabla v_{h}\right)_{\Omega \cap \hat{\Omega}}+\frac{1}{\epsilon}\left(\nabla\left(u_{\epsilon, h}-\hat{u}_{\epsilon, h}\right), \nabla v_{h}\right)_{\Omega \cap \hat{\Omega}_{1}}+\left(\nabla u_{\epsilon, h}, \nabla v_{h}\right)_{\Omega \backslash \hat{\Omega}} \\
+ & \frac{1}{\epsilon}\left(\nabla u_{\epsilon, h}, \nabla v_{h}\right)_{\Omega_{1} \backslash \hat{\Omega}_{1}}-\left(\nabla \hat{u}_{\epsilon, h}, \nabla v_{h}\right)_{\hat{\Omega} \backslash \Omega}-\frac{1}{\epsilon}\left(\nabla \hat{u}_{\epsilon, h}, \nabla v_{h}\right)_{\hat{\Omega}_{1} \backslash \Omega_{1}} \\
= & \left(\tilde{f}-f_{h}, v_{h}\right)_{D}, \forall v_{h} \in V_{h} .
\end{aligned}
$$

Since $\Omega_{1} \backslash \hat{\Omega}_{1}=\hat{\Omega} \backslash \Omega$ and $\hat{\Omega}_{1} \backslash \Omega_{1}=\Omega \backslash \hat{\Omega}$, the above equation can be written as

$$
\begin{aligned}
& \left(\nabla\left(u_{\epsilon, h}-\hat{u}_{\epsilon, h}\right), \nabla v_{h}\right)_{\Omega \cup \hat{\Omega}}+\frac{1}{\epsilon}\left(\nabla\left(u_{\epsilon, h}-\hat{u}_{\epsilon, h}\right), \nabla v_{h}\right)_{\Omega \cap \hat{\Omega}_{1}} \\
= & \left(\frac{1}{\epsilon}-1\right)\left(\nabla \hat{u}_{\epsilon, h}, \nabla v_{h}\right)_{\hat{\Omega}_{1} \backslash \Omega_{1}}+\left(1-\frac{1}{\epsilon}\right)\left(\nabla u_{\epsilon, h}, \nabla v_{h}\right)_{\Omega_{1} \backslash \hat{\Omega}_{1}}+\left(\tilde{f}-f_{h}, v_{h}\right)_{D} .
\end{aligned}
$$

We apply Lemma 5.1 to obtain

$$
\begin{aligned}
& \left|\hat{u}_{\epsilon, h}\right|_{1, \hat{\Omega}_{1} \backslash \Omega_{1}} \leq C \sqrt{h}\left|\hat{u}_{\epsilon, h}\right|_{1, \hat{\Omega}_{1}} \leq C \sqrt{h} \epsilon, \\
& \left|v_{h}\right|_{1, \hat{\Omega}_{1} \backslash \Omega_{1}} \leq C \sqrt{h}\left|v_{h}\right|_{1, \hat{\Omega}_{1}}\left(\text { or } \sqrt{h}\left|v_{h}\right|_{1, \Omega}\right), \\
& \left|u_{\epsilon, h}\right|_{1, \Omega_{1} \backslash \hat{\Omega}_{1}} \leq C \sqrt{h}\left|u_{\epsilon, h}\right|_{1, \Omega_{1}} \leq C \sqrt{h} \epsilon, \\
& \left|v_{h}\right|_{1, \Omega_{1} \backslash \hat{\Omega}_{1}} \leq C \sqrt{h}\left|v_{h}\right|_{1, \Omega_{1}}\left(\text { or } \sqrt{h}\left|v_{h}\right|_{1, \hat{\Omega}}\right) .
\end{aligned}
$$


Since we have $\left\|\tilde{f}-f_{h}\right\|_{0, D} \leq C h$, for $v_{h}=u_{\epsilon, h}-\hat{u}_{\epsilon, h}$, we get

$$
\begin{aligned}
& \left|u_{\epsilon, h}-\hat{u}_{\epsilon, h}\right|_{1, \Omega \cup \hat{\Omega}}^{2}+\frac{1}{\epsilon}\left|u_{\epsilon, h}-\hat{u}_{\epsilon, h}\right|_{1, \Omega_{1} \cap \hat{\Omega}_{1}}^{2} \\
\leq & C h\left(\left|u_{\epsilon, h}-\hat{u}_{\epsilon, h}\right|_{1, \Omega \cup \hat{\Omega}}+\left\|u_{\epsilon, h}-\hat{u}_{\epsilon, h}\right\|_{0, D}\right) .
\end{aligned}
$$

This, together with the Poincaré inequality, implies the desired result.

5.2 Neumann boundary value problem

$\left(\hat{Q}_{\epsilon, h}\right)$ reads as:

$$
\left\{\begin{array}{l}
\text { Find } \hat{u}_{\epsilon, h} \in V_{h}(D) \text { such that } \\
\left(\hat{u}_{\epsilon, h}, v_{h}\right)_{1, \hat{\Omega}}+\epsilon\left(\nabla \hat{u}_{\epsilon, h}, \nabla v_{h}\right)_{\hat{\Omega}_{1}}=\left(f_{h}, v_{h}\right)_{D}, \quad \forall v_{h} \in V_{h}(D) .
\end{array}\right.
$$

Lemma $5.3(\Omega \backslash \hat{\Omega}) \cup(\hat{\Omega} \backslash \Omega) \subset \Gamma_{\delta}, \delta=O\left(h^{2}\right)$ implies that:

$$
\begin{aligned}
&\left\|v_{h}\right\|_{0, \Omega \backslash \hat{\Omega}} \leq C h\left\|v_{h}\right\|_{1, \Omega}\left(\text { or } h\left\|v_{h}\right\|_{1, \hat{\Omega}_{1}}\right) . \\
&\left\|v_{h}\right\|_{0, \hat{\Omega} \backslash \Omega} \leq C h\left\|v_{h}\right\|_{1, \hat{\Omega}}\left(\text { or } h\left\|v_{h}\right\|_{1, \Omega_{1}}\right) .
\end{aligned}
$$

(This lemma is quoted form [10].)

Theorem 5.4 There exists a unique solution $\hat{u}_{\epsilon, h}$ for (5.4). If $\tilde{f}-f_{h}=0$ on $\Omega_{1} \cap \hat{\Omega}_{1}$, and $u_{\epsilon, h}$ is the solution of (4.12), then we have,

$$
\left\|u_{\epsilon, h}-\hat{u}_{\epsilon, h}\right\|_{1, \Omega \cup \hat{\Omega}} \leq C h .
$$

The proof is an analogue of that of Theorem 5.2, with using Lemmas 5.1 and 5.3.

\section{Numerical experiments}

Let $\tilde{u} \in H_{0}^{1}(D)$ be the zero extension of the solution $u \in H_{0}^{1}(\Omega)$ of the Dirichlet boundary value problem (1.1). Then, from the results of previous sections, we find that

$$
\begin{aligned}
& \left\|\hat{u}_{\epsilon, h}-\tilde{u}\right\|_{1, D} \leq\left\|\hat{u}_{\epsilon, h}-u_{\epsilon, h}\right\|_{1, D}+\left\|u_{\epsilon, h}-u_{\epsilon}\right\|_{1, D}+\left\|u_{\epsilon}-\tilde{u}\right\|_{1, D} \\
= & \left\|\hat{u}_{\epsilon, h}-u_{\epsilon, h}\right\|_{1, D}+\left\|u_{\epsilon, h}-u_{\epsilon}\right\|_{1, D}+\left\|\left.u_{\epsilon}\right|_{\Omega}-u\right\|_{1, \Omega}+\left\|u_{\epsilon}\right\|_{1, \Omega_{1}} \\
\leq & C h+C(\sqrt{h}+\sqrt{\epsilon})+C \epsilon+C \epsilon \\
\leq & C(\sqrt{h}+\sqrt{\epsilon}) .
\end{aligned}
$$

In addition, for the error in $L^{2}$ norm, we also have

$$
\left\|\hat{u}_{\epsilon, h}-\tilde{u}\right\|_{0, D} \leq C(\epsilon+h+\sqrt{h \epsilon}) .
$$


Fig. $4 \Omega, D$ and the mesh
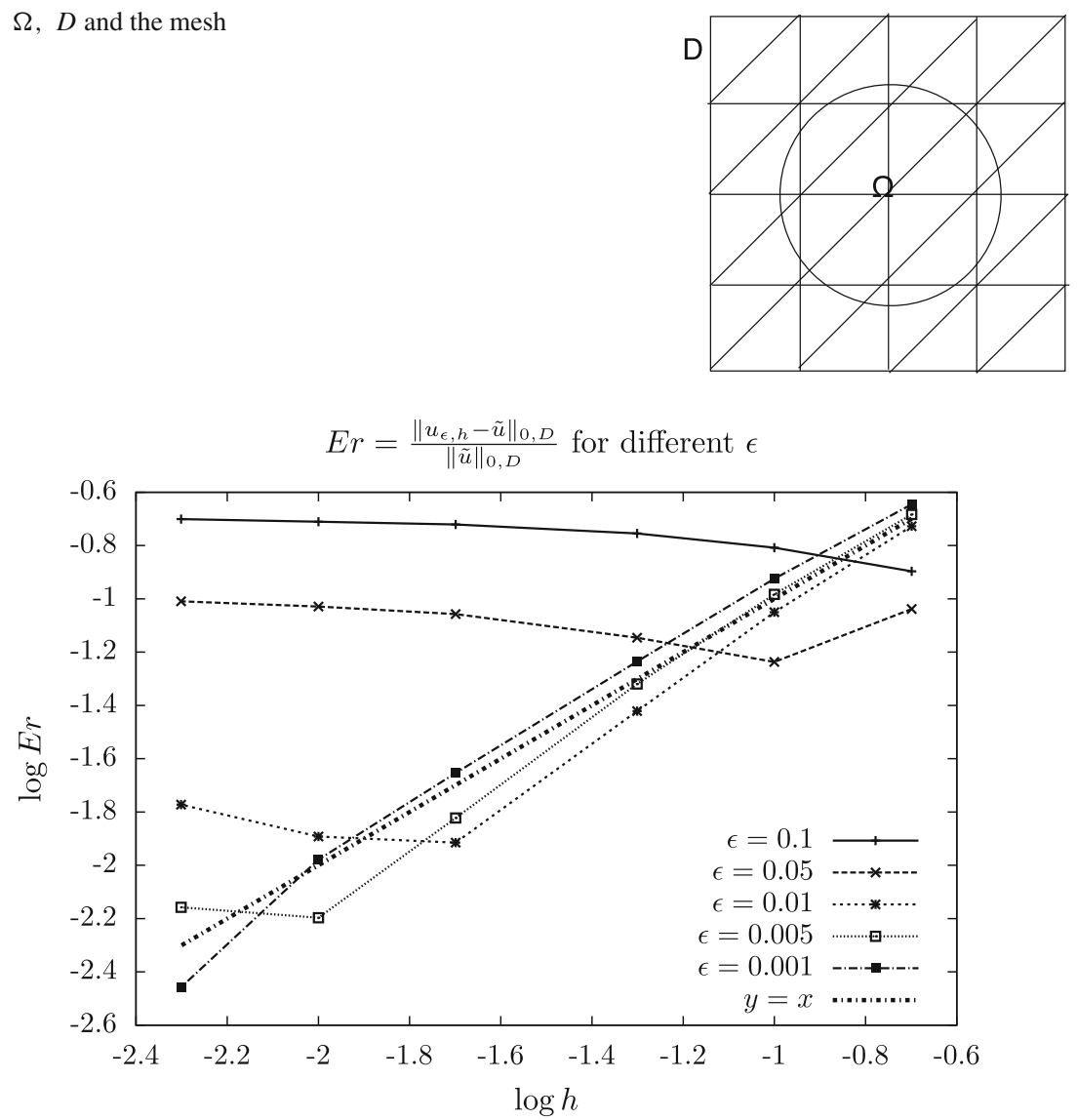

Fig. $5 \frac{\left\|u_{\epsilon, h}-\tilde{u}\right\|_{0, D}}{\|\tilde{u}\|_{0, D}}$ with different $\epsilon$ and $h$

So in our computation, to calculate $L^{2}$ and $H^{1}$ errors of $\hat{u}_{\epsilon, h}-\tilde{u}$ on D is sufficient to verify the theoretical results, which is more practical than computing the norm of $\left.\hat{u}_{\epsilon, h}\right|_{\Omega}-u$ in $\Omega$, because of the curved boundary of $\Omega$. Now, let $\Omega=\left\{(x, y) \in \mathbb{R}^{2} \mid\right.$ $\left.x^{2}+y^{2}<4\right\}$. The original problem reads as:

$$
\left\{\begin{array}{l}
-\Delta u=4 \text { in } \Omega \\
u=0 \text { on } \Gamma .
\end{array}\right.
$$

The exact solution is $u=4-x^{2}-y^{2}$. Let $D=\{(x, y) \mid-3<x<3,-3<y<$ 3\}, $\Omega \subset D$, and generate an $N \times N$ grid in the square $D$ as the Cartesian mesh (see Fig. 4). For $N=30,60,120,300,600,1200$, setting $h=\frac{6}{N}, \sqrt{2} h$ is the diameter of the triangles.

The error estimates are showed in Figs. 5 and 6 with the logarithm (log) to base 10, from which we see that for fixed $\epsilon, L^{2}$ error behaves as $C h$ (in Fig. 5, $\frac{\log E r}{\log h} \approx 1$ ) and $H^{1}$ error behaves as $C \sqrt{h}$ (in Fig. $6, \frac{\log E r}{\log h} \approx \frac{1}{2}$ ). But at the same time, they 


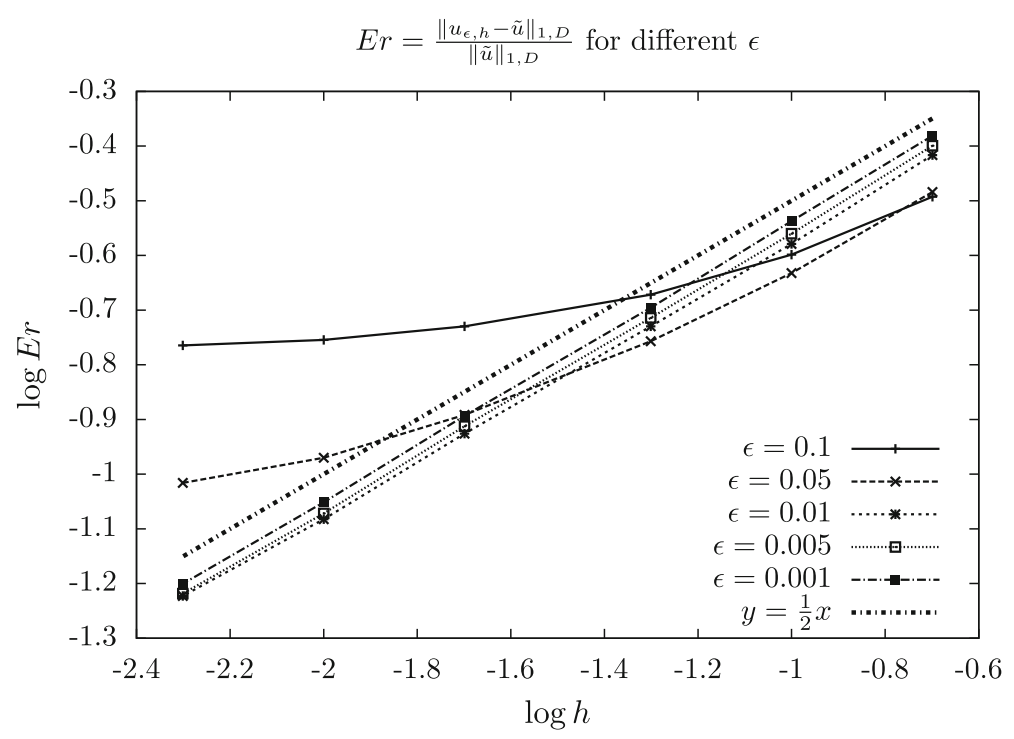

Fig. $6 \frac{\left\|u_{\epsilon, h}-\tilde{u}\right\|_{1, D}}{\|\tilde{u}\|_{1, D}}$ with different $\epsilon$ and $h$

also have lower bounds even if we allow $h$ to become arbitrarily small, since the error estimates are also bounded by $\epsilon$, according to (6.1) and (6.2). And we can observe that, for different $\epsilon, L^{2}$ error has the lower bound approximate to $C \epsilon$, and $H^{1}$ error is bounded by $C \sqrt{\epsilon}$. This confirms our theoretical results.

\section{References}

1. Chen, Z., Zou, J.: Finite element methods and their convergence for elliptic and parabolic interface problems. Numer. Math. 79(2), 175202 (1998)

2. Del Pino, S., Pironneau, O.: A fictitious domain based general PDE solver. In: Numerical Methods for Scientific Computing. Variational Problems and Applications, 2030, International Center Numeric Methods and Engineering (CIMNE), Barcelona (2003)

3. Grisvard, P.: Elliptic problems in nonsmooth domains. In: Monographs and Studies in Mathematics, vol. 24. Pitman (Advanced Publishing Program), Boston, MA (1985)

4. Grisvard, P.: Singularities in boundary value problems Recherches en Mathématiques Appliquées [Research in Applied Mathematics], vol. 22. Springer-Verlag, Berlin (1992)

5. Lions, J.L., Magenes, E.: Non-Homogeneous Boundary Value Problems and Applications, vol. I. Springer-Verlag, New York (1972)

6. Lions, J.L.: Une remarque sur les problémes d'évolution non linéaires dans des domaines non cylindriques, Rev. Roumaine Math. Pures Appl. 9, 1118 (1964)

7. Mignot, A.L.: Méthodes d'approximation des solutions de certains problèmes aux limites linéaires. I. (French) Rend. Sem. Mat. Univ. Padova 40, 1138 (1968)

8. Maury, B.: Numerical analysis of a finite element/volume penalty method. SIAM J. Numer. Anal. 47(2), 11261148 (2009)

9. Ohmori, K., Saito, N.: On the convergence of finite element solutions to the interface problem for the Stokes system. J. Comput. Appl. Math. 198(1), 116128 (2007)

10. Tabata, M.: Uniform solvability of finite element solutions in approximate domains. Jpn. J. Indust. Appl. Math. 18(2), 567585 (2001) 
11. Zhang, S.: A domain embedding method for mixed boundary value problems. C. R. Math. Acad. Sci. Paris 343(4), 287290 (2006)

12. Zhang, S.: Analysis of finite element domain embedding methods for curved domains using uniform grids, SIAM J. Numer. Anal. 46(6), 28432866 (2008)

13. Z̃enís̃ek, A.: Nonlinear elliptic and evolution problems and their finite element approximations. In: Computational Mathematics and Applications. Academic Press, Inc. [Harcourt Brace Jovanovich, Publishers], London (1990)

14. Zhou, G.: Analysis of the Fictitious Domain Method with $H^{1}$-Penalty for Parabolic Problem. submitted, UTMS preprint series 2012-9

15. Zhou, G.: Analysis of the Fictitious Domain Method with $L^{2}$-Penalty for Elliptic and Parabolic Problems. submitted, UTMS preprint series 2012-18 